\title{
Excitation functions of parameters in Erlang distribution, Schwinger mechanism, and Tsallis statistics in RHIC BES program
}

\author{
$\mathrm{Li}-\mathrm{Na} \mathrm{Gao}^{a}, \mathrm{Fu}-\mathrm{Hu} \mathrm{Liu}^{a}, 1$, and Roy A. Lacey ${ }^{b}$. \\ ${ }^{a}$ Institute of Theoretical Physics, Shanxi University, Taiyuan, Shanxi 030006, China \\ ${ }^{b}$ Departments of Chemistry 8 Physics, Stony Brook University, Stony Brook, NY 11794, USA
}

\begin{abstract}
Experimental results of the transverse momentum distributions of $\phi$ mesons and $\Omega$ hyperons produced in gold-gold $(\mathrm{Au}-\mathrm{Au})$ collisions with different centrality intervals, measured by the STAR Collaboration at different energies $(7.7,11.5,19.6,27$, and $39 \mathrm{GeV}$ ) in the beam energy scan (BES) program at the relativistic heavy ion collider (RHIC), are approximately described by the single Erlang distribution and the two-component Schwinger mechanism. Meanwhile, the STAR experimental transverse momentum distributions of negatively charged particles, produced in Au-Au collisions at RHIC BES energies, are approximately described by the two-component Erlang distribution and the single Tsallis statistics. The excitation functions of free parameters are obtained from the fit to the experimental data. A weak softest point in the string tension in $\Omega$ hyperon spectra is observed at $7.7 \mathrm{GeV}$.
\end{abstract}

Keywords: Transverse momentum distribution, excitation functions of parameters, Erlang distribution, Schwinger mechanism, Tsallis statistics

PACS: 12.38.Mh, 25.75.Dw, 24.10.Pa

\section{Introduction}

High energy nucleus-nucleus (heavy ion) collisions at the relativistic heavy ion collider (RHIC) [1-3] and the large hadron collider ( $\mathrm{LHC}$ ) $[4,5]$ can provide the environment and condition of high temperature and density, where a new state of matter, namely the quark-gluon plasma (QGP), is expected to form [6-8]. Even at the top center-of-mass energy (the maximum $\sqrt{s_{N N}}=19.4 \mathrm{GeV}$ ) of the super proton synchrotron (SPS), the equation of state (EoS) is possibly different from that at lower energy [9]. Both the RHIC and SPS have performed the beam energy scan (BES) programs, which give us opportunities to search for the onset of deconfinement of quarks and gluons, the critical point of phase transition from QGP to hadronic matter, and the softest points in the EoS in nucleus-nucleus collisions. The onset of quark deconfinement should be possibly the critical point of QGP phase transition. Although there is a relation between the softest points in the EoS and the critical point of QGP phase transition, they are not sure the same. In addition, different quantities result in different softest points, and the critical point should be exclusive. To reveal the relation between the softest points and critical point is still an open question.

Generally, the RHIC and its BES energies $\left(\sqrt{s_{N N}}=7.7-200 \mathrm{GeV}\right)[10-12]$ are larger than the SPS and its BES energies $\left(\sqrt{s_{N N}}=6.6-19.4 \mathrm{GeV}\right)[13-15]$, though they have an overlap each other. The STAR Collaboration has been performing the RHIC BES program [10-12], and the NA61/SHINE Collaboration has been performing the SPS BES program [13-15]. Some interesting results involved to the softest points in some excitation functions in the EoS have been reported. For example, the works which study excitation functions of ratio of positive kaon number to pions $\left(K^{+} / \pi^{+}\right)$[16-18], chemical freeze-out temperature $\left(T_{c h}\right)[17,18]$, mean transverse mass minus rest mass $\left(\left\langle m_{T}\right\rangle-m_{0}\right)$ [17], and ratio of experimental negative pion rapidity $(y)$ distribution width to Landau hydrodynamic model prediction $\left[\sigma_{y}\left(\pi^{-}\right) / \sigma_{y}\right.$ (hydro)] [18] show the softest point being around $\sqrt{s_{N N}}=7-8 \mathrm{GeV}$. By using the Landau hydrodynamic model

\footnotetext{
${ }^{1}$ E-mail: fuhuliu@163.com; fuhuliu@sxu.edu.cn

${ }^{2}$ E-mail: Roy.Lacey@Stonybrook.edu
} 
and the ultra-relativistic quantum molecular dynamics hybrid approach, an analysis based on rapidity distribution and squared speed-of-sound $\left(c_{s}^{2}\right)$ extraction shows the softest point locating in the energy range from 4 to $9 \mathrm{GeV}[19,20]$.

Recently, a wiggle in the excitation function of a specific reduced curvature, $C_{y}$, of the net-proton rapidity distribution at midrapidity is expected in the energy range from 4 to $8 \mathrm{GeV}[21,22]$, where $C_{y}=\left(y_{\text {beam }}^{3} d^{3} N / d y^{3}\right)_{y=0} /\left(y_{\text {beam }} d N / d y\right)_{y=0}, y_{\text {beam }}$ denotes the beam rapidity, and $N$ denotes the number of considered particles. A local weak wiggle in the $C_{y}$ excitation function is exhibited in the range from 8 to $12 \mathrm{GeV}$. It is shown that there are more than one softest points in the EoS from 4 to $12 \mathrm{GeV}$. Our recent work which based on rapidity distribution and $c_{s}^{2}$ extraction shows the softest point being around $8.8 \mathrm{GeV}$ [23], and there is a jump (from $\leq 0.3$ to $1 / 3-1 / 2$ ) in $c_{s}^{2}$ when the energy changes from 17.3 to $19.6 \mathrm{GeV}$. These phenomenons render that the interacting process is complex in the considered energy range. However, the searching for the onset of quark deconfinement and the critical point of QGP phase transition is not simple [24]. More analyses are needed in the study of the relation between the softest points and critical point.

The present work does not intend to reveal the relation between the softest points in the EoS and the critical points of QGP phase transition. Instead, we hope to search for more information of the softest points. In this paper, we study the transverse momentum spectra of different particles $(\phi$ mesons, $\Omega$ hyperons, and negatively charged particles) produced in gold-gold ( $\mathrm{Au}-\mathrm{Au}$ ) collisions at a few RHIC BES energies $(7.7,11.5,19.6,27$, and $39 \mathrm{GeV}$ ) by using the single (or two-component) Erlang distribution [25-27] for both hard and soft processes, two-component Schwinger mechanism [28-31] for hard process only, and single Tsallis statistics [32-34] for soft process only. The results calculated by the two methods (Schwinger and Erlang, or Tsallis and Erlang) are compared with the experimental data of the STAR Collaboration $[35,36]$.

The structure of the present work is as follows. The model and method are shortly described in section 2. Results and discussion are given in section 3. In section 4, we summarize our main observations and conclusions.

\section{The model and method}

Firstly, we discuss uniformly hard and soft collision processes in the framework of the multisource thermal model [25-27]. According to the model, a given particle is produced in the collision process where a few partons taken part in. Hard process contains two or three partons which are valence quarks. Soft process contains usually two or more partons which are gluons and sea quarks. Each (the $i$-th) parton is assumed to contribute to an exponential function $\left[f_{i}\left(p_{t}\right)\right]$ of transverse momentum $\left(p_{t}\right)$ distribution. Let $\left\langle p_{t}\right\rangle$ denotes the mean transverse momentum contributed by each (the $i$-th) parton, we have

$$
f_{i}\left(p_{t}\right)=\frac{1}{\left\langle p_{t}\right\rangle} \exp \left(-\frac{p_{t}}{\left\langle p_{t}\right\rangle}\right)
$$

The contribution of all $n$ partons which taken part in the collision process is the folding of $n$ exponential functions [25-27]. We have the transverse momentum $\left(p_{T}\right)$ distribution $f\left(p_{T}\right)$ of final-state particles to be the Erlang distribution

$$
f\left(p_{T}\right)=\frac{p_{T}^{n-1}}{(n-1) !\left\langle p_{t}\right\rangle^{n}} \exp \left(-\frac{p_{T}}{\left\langle p_{t}\right\rangle}\right)
$$

which has the mean transverse momentum $\left\langle p_{T}\right\rangle=n\left\langle p_{t}\right\rangle$.

Secondly, the production of particles with heavy mass in hard process is also described by the Schwinger mechanism [28-31]. Let $\kappa$ denote the string tension between partons which form a given final-state particle. Each (the $i$-th) parton has and then contributes to the given particle a Gaussian function of transverse momentum distribution

$$
f_{i}\left(p_{t}\right)=\frac{1}{\sqrt{\kappa}} \exp \left(-\frac{\pi p_{t}^{2}}{\kappa}\right)
$$

The transverse momentum distribution contributed by the string (two partons) is the folding of two 
Gaussian functions. That is

$$
f\left(p_{T}\right)=\int_{0}^{p_{T}} f_{1}\left(p_{t}\right) f_{2}\left(p_{T}-p_{t}\right) d p_{t}=\frac{1}{\kappa} \int_{0}^{p_{T}} \exp \left\{-\frac{\pi\left[p_{t}^{2}+\left(p_{T}-p_{t}\right)^{2}\right]}{\kappa}\right\} d p_{t} .
$$

Thirdly, the Tsallis statistics which has more than one function forms [32-34] is also used to describe the thermal production of particles in soft process. We consider the simplest form of the Tsallis transverse momentum distribution at mid-rapidity

$$
f\left(p_{T}\right)=C_{T} p_{T} \sqrt{p_{T}^{2}+m_{0}^{2}}\left[1+\frac{q-1}{T}\left(\sqrt{p_{T}^{2}+m_{0}^{2}}-\mu\right)\right]^{-q /(q-1)},
$$

where $T$ is the temperature which describes averagely a few local sources (equilibrium states), $q$ is the entropy index which describes the degree of non-equilibrium among different local sources, $\mu$ is the chemical potential which is related to $\sqrt{s_{N N}}[37], m_{0}$ is the rest mass of the considered particle, and $C_{T}$ is the normalization constant which is related to other parameters.

In the Monte Carlo method, let $r_{i}$ denote random numbers in $[0,1]$. The Erlang distribution results in

$$
p_{T}=-\left\langle p_{t}\right\rangle \sum_{i=1}^{n} \ln r_{i}=-\left\langle p_{t}\right\rangle \ln \prod_{i=1}^{n} r_{i} .
$$

Although both $-\left\langle p_{t}\right\rangle \sum_{i=1}^{n} \ln r_{i}$ and $-\left\langle p_{t}\right\rangle \ln \prod_{i=1}^{n} r_{i}$ can be used, we would rather use $-\left\langle p_{t}\right\rangle \sum_{i=1}^{n} \ln r_{i}$ than $-\left\langle p_{t}\right\rangle \ln \prod_{i=1}^{n} r_{i}$ due to $\prod_{i=1}^{n} r_{i}$ being probably a too small value for the calculation when $n$ is large enough. Similarly, let $R_{1,2,3,4,5}$ denote random numbers in $[0,1]$. The Schwinger mechanism results in

$$
p_{T}=\sqrt{\frac{\kappa}{\pi}}\left[\sqrt{-\ln R_{1}} \cos \left(2 \pi R_{2}\right)+\sqrt{-\ln R_{3}} \cos \left(2 \pi R_{4}\right)\right] .
$$

The Tsallis distribution satisfies

$$
\int_{0}^{p_{T}} f\left(p_{T}\right) d p_{T}<R_{5}<\int_{0}^{p_{T}+d p_{T}} f\left(p_{T}\right) d p_{T} .
$$

Sometimes, the experimental $p_{T}$ distribution is the sum of two components with different weights. This means that we need the two-component function in some cases. Although we show the Monte Carlo method of the calculations, the analytical method is used in the present work, unless $(n-1)$ ! is too large for the calculations.

It should be noted that the Erlang distribution shows flexible forms which are the foldings of two, three, $\cdots$, or multiple exponential functions, the Schwinger mechanism shows an inflexible form which is the folding of two Gaussian functions, while the Tsallis statistics shows relative flexible form which is the sum of two or three standard distributions [38]. The parameter $\left\langle p_{t}\right\rangle$ in the Erlang distribution renders the mean transverse momentum contributed by each parton, the parameter $\kappa$ in the Schwinger mechanism renders the string tension between two collision partons, while the parameter $T$ in the Tsallis statistics renders the excitation degree of interacting system. Because of the parton number in a string being fixed to two, the Schwinger mechanism is not suitable in analysis of soft process in which there are two or more partons, unless we consider multiple strings and the folding of multiple Gaussian functions in the process. In the case of using two or more components, the parameter value can be obtained by the average weighted those of different components. As a thermal description, the Tsallis statistics is not suitable in analysis of hard process in which heavy particles are produced by direct collisions between two or among three partons.

\section{Results and discussion}

Figure 1 presents the transverse momentum distributions, $d^{2} N /\left(2 \pi N_{e v t} p_{T} d y d p_{T}\right)$, of $\phi$ mesons produced in mid-rapidity $(|y|<0.5)$ in Au-Au collisions at five RHIC BES energies: (a) 7.7, (b) 11.5, (c) 19.6, (d) 27, and (e) $39 \mathrm{GeV}$, where $N_{\text {evt }}$ denotes the event number and $\phi$ mesons are assumed to produce 


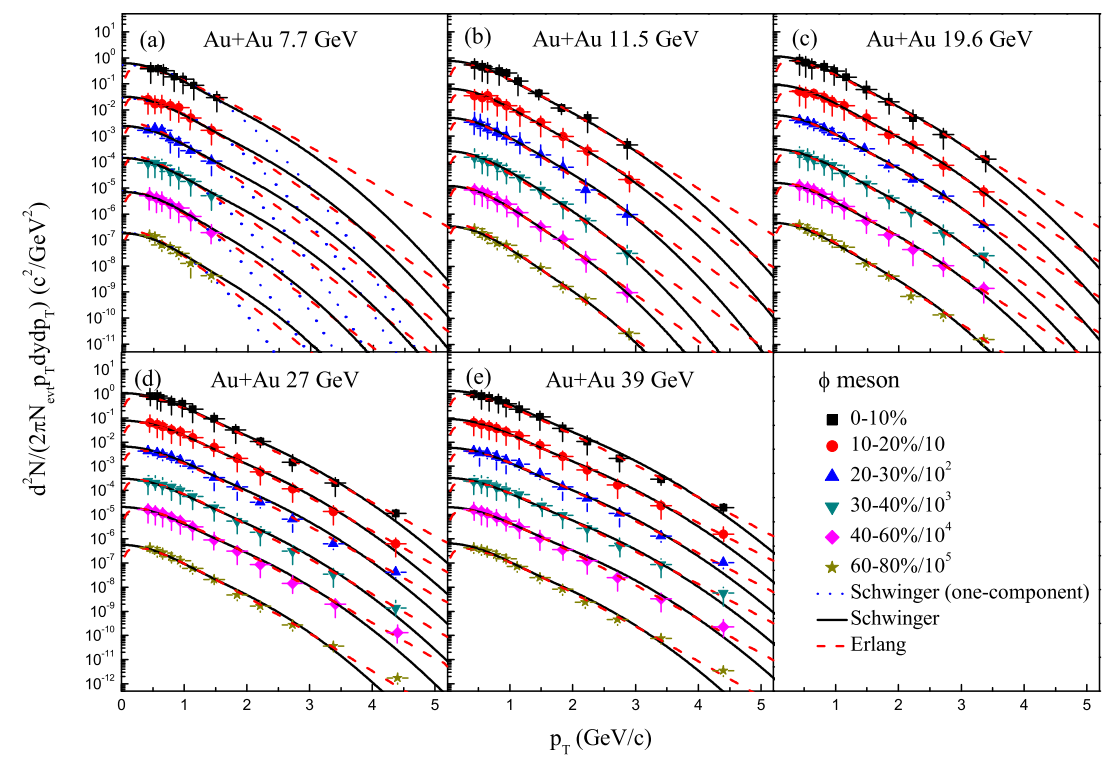

Figure 1. Transverse momentum distributions of $\phi$ mesons produced in $|y|<0.5$ in $\mathrm{Au}-\mathrm{Au}$ collisions at five RHIC BES energies: (a) 7.7, (b) 11.5, (c) 19.6, (d) 27, and (e) $39 \mathrm{GeV}$. The symbols represent the experimental data of the STAR Collaboration [35] in different centrality intervals, which are scaled by different amounts marked in the panel. The dashed and solid curves are our results calculated by using the single Erlang distribution and the two-component Schwinger mechanism respectively. The dotted curves in Figure 1(a) are an example calculated by using the single (one-component) Schwinger mechanism.

in hard process. The symbols represent the experimental data of the STAR Collaboration [35] in different centrality intervals, which are scaled by different amounts marked in the panel. The dashed and solid curves are our results calculated by using the single Erlang distribution and the two-component Schwinger mechanism respectively. The values of $n$ for all the cases are taken to be 3 . Other related parameter values are listed in Table 1 with values of $\chi^{2}$ per degree of freedom $\left(\chi^{2} /\right.$ dof $)$, where $k_{S 1}$ denotes the relative contribution of the first component in the two-component Schwinger mechanism, and $\kappa_{1}, \kappa_{2}$, and $\left\langle p_{t}\right\rangle$, are the main free parameters in the two functions. One can see that both the single Erlang distribution and the two-component Schwinger mechanism describe approximately the experimental transverse momentum distributions of $\phi$ mesons produced in mid-rapidity in $\mathrm{Au}-\mathrm{Au}$ collisions at the RHIC BES in most cases.

We would like to point out that the experimental data in narrow transverse momentum range should be described by the one-component formula of the Schwinger mechanism. As an example, the dotted curves in Figure 1(a) are the results of the single Schwinger mechanism with $\kappa=1.462 \pm 0.283,1.435 \pm 0.275$, $1.300 \pm 0.186,1.156 \pm 0.169,1.070 \pm 0.147$, and $0.992 \pm 0.163 \mathrm{GeV} / \mathrm{fm}$, resulting in $\chi^{2} / \mathrm{dof}=0.084,0.123$, $0.200,0.056,0.044$, and 0.421 , respectively, when the centrality interval changes from $0-10 \%$ to $60-80 \%$. Although less parameters are used in the fit of one-component formula, it does not describe the data in wide transverse momentum range.

Figure 2 is similar to Figure 1, but it shows the results, $d^{2} N /\left(2 \pi N_{e v t} p_{T} d y d p_{T}\right)$, of $\Omega^{-}$(solid symbols) and $\bar{\Omega}^{+}$(open symbols divided by 2 ) in different centrality intervals, where the symbols represent the data of the STAR Collaboration [35] and $\Omega$ hyperons are assumed to produce in hard process. At the same time, the result of the one-component Schwinger mechanism is not available. The values of $n$ for all the cases are taken to be 3 . Other related parameters are listed in Table 2 with values of $\chi^{2} /$ dof. One can see that both the single Erlang distribution and the two-component Schwinger mechanism describe approximately the experimental data of $\Omega^{-}$and $\bar{\Omega}^{+}$produced in mid-rapidity region in Au-Au collisions at the RHIC BES in most cases. 


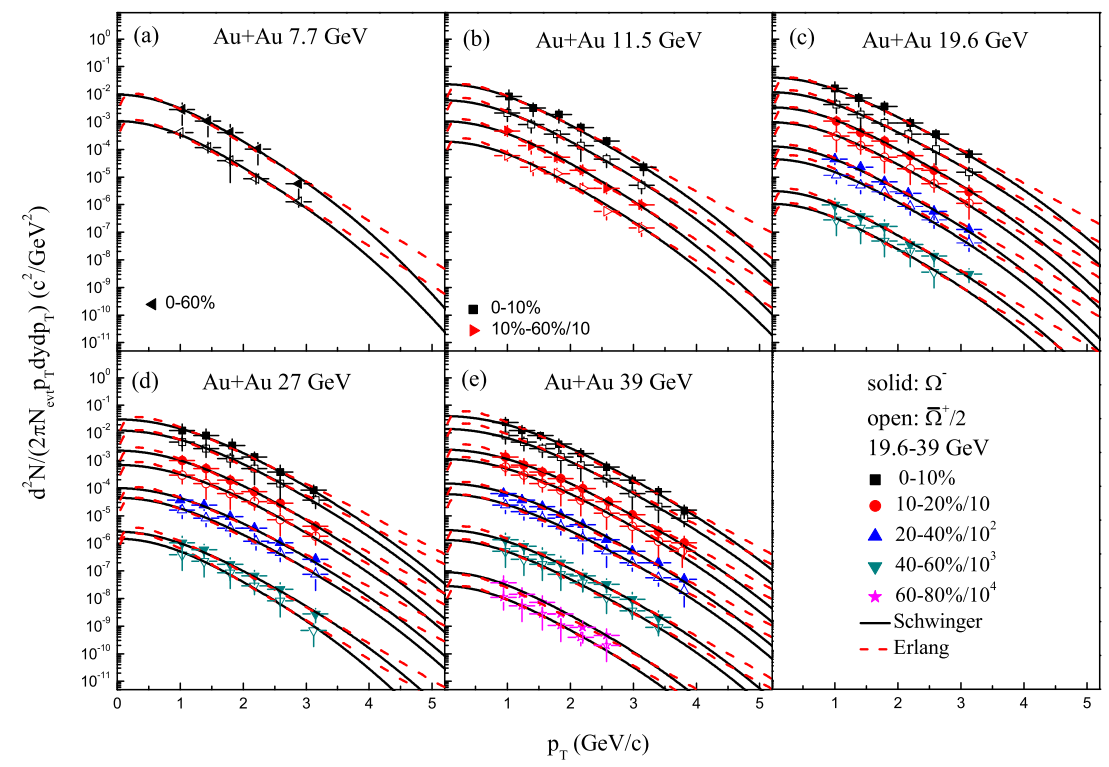

Figure 2. The same as for Fig. 1, but showing the results of $\Omega^{-}$(solid symbols) and $\bar{\Omega}^{+}$(open symbols divided by 2) in different centrality intervals, where the symbols represent the data of the STAR Collaboration [35]. At the same time, the results of the one-component Schwinger mechanism are not available.

Table 1. Values of parameters $k_{S 1}, \kappa_{1}, \kappa_{2}$, and $\left\langle p_{t}\right\rangle$, as well as $\chi^{2} /$ dof corresponding to the solid and dashed curves in Fig. 1. In all the cases, for the single Erlang distribution, we have $n=3$ which are not listed in the column.

\begin{tabular}{|c|c|c|c|c|c|c|c|}
\hline \multirow[b]{2}{*}{ Figure } & \multirow[b]{2}{*}{ Type } & \multicolumn{4}{|c|}{ Two-component Schwinger mechanism } & \multicolumn{2}{|c|}{ Single Erlang distribution } \\
\hline & & $k_{S 1}$ & $\kappa_{1}(\mathrm{GeV} / \mathrm{fm})$ & $\kappa_{2}(\mathrm{GeV} / \mathrm{fm})$ & $\chi^{2} / \mathrm{dof}$ & $\left\langle p_{t}\right\rangle(\mathrm{GeV} / c)$ & $\chi^{2} /$ dof \\
\hline \multirow[t]{6}{*}{ Figure 1(a) } & $0-10 \%$ & $0.628 \pm 0.135$ & $0.967 \pm 0.132$ & $2.635 \pm 0.245$ & 0.050 & $0.287 \pm 0.057$ & 0.076 \\
\hline & $10-20 \%$ & $0.620 \pm 0.133$ & $0.952 \pm 0.127$ & $2.598 \pm 0.228$ & 0.120 & $0.247 \pm 0.046$ & 0.160 \\
\hline & $20-30 \%$ & $0.615 \pm 0.137$ & $0.936 \pm 0.133$ & $2.564 \pm 0.237$ & 0.225 & $0.231 \pm 0.043$ & 0.288 \\
\hline & $30-40 \%$ & $0.612 \pm 0.132$ & $0.895 \pm 0.135$ & $2.473 \pm 0.238$ & 0.066 & $0.230 \pm 0.038$ & 0.185 \\
\hline & $40-60 \%$ & $0.605 \pm 0.128$ & $0.862 \pm 0.132$ & $2.126 \pm 0.219$ & 0.066 & $0.228 \pm 0.040$ & 0.092 \\
\hline & $60-80 \%$ & $0.596 \pm 0.133$ & $0.787 \pm 0.137$ & $1.985 \pm 0.225$ & 0.364 & $0.208 \pm 0.038$ & 0.116 \\
\hline \multirow[t]{6}{*}{ Figure 1(b) } & $0-10 \%$ & $0.632 \pm 0.132$ & $0.988 \pm 0.306$ & $2.682 \pm 0.382$ & 0.269 & $0.273 \pm 0.037$ & 0.516 \\
\hline & $10-20 \%$ & $0.624 \pm 0.124$ & $0.953 \pm 0.313$ & $2.500 \pm 0.375$ & 0.096 & $0.267 \pm 0.033$ & 0.183 \\
\hline & $20-30 \%$ & $0.618 \pm 0.108$ & $0.922 \pm 0.298$ & $2.266 \pm 0.323$ & 0.113 & $0.247 \pm 0.033$ & 0.135 \\
\hline & $30-40 \%$ & $0.613 \pm 0.115$ & $0.892 \pm 0.303$ & $2.000 \pm 0.337$ & 0.020 & $0.238 \pm 0.035$ & 0.152 \\
\hline & $40-60 \%$ & $0.600 \pm 0.113$ & $0.870 \pm 0.312$ & $1.892 \pm 0.357$ & 0.063 & $0.228 \pm 0.032$ & 0.200 \\
\hline & $60-80 \%$ & $0.588 \pm 0.122$ & $0.823 \pm 0.297$ & $1.870 \pm 0.345$ & 0.214 & $0.218 \pm 0.035$ & 0.375 \\
\hline \multirow[t]{6}{*}{ Figure $1(\mathrm{c})$} & $0-10 \%$ & $0.635 \pm 0.157$ & $1.092 \pm 0.272$ & $2.735 \pm 0.336$ & 0.112 & $0.278 \pm 0.042$ & 0.186 \\
\hline & $10-20 \%$ & $0.626 \pm 0.148$ & $0.976 \pm 0.304$ & $2.642 \pm 0.358$ & 0.115 & $0.273 \pm 0.036$ & 0.525 \\
\hline & $20-30 \%$ & $0.610 \pm 0.153$ & $0.952 \pm 0.285$ & $2.553 \pm 0.326$ & 0.129 & $0.262 \pm 0.032$ & 0.093 \\
\hline & $30-40 \%$ & $0.602 \pm 0.155$ & $0.938 \pm 0.287$ & $2.493 \pm 0.347$ & 0.132 & $0.260 \pm 0.035$ & 0.083 \\
\hline & $40-60 \%$ & $0.593 \pm 0.157$ & $0.923 \pm 0.257$ & $2.456 \pm 0.334$ & 0.967 & $0.258 \pm 0.033$ & 0.121 \\
\hline & $60-80 \%$ & $0.590 \pm 0.152$ & $0.832 \pm 0.232$ & $2.180 \pm 0.305$ & 0.364 & $0.242 \pm 0.038$ & 0.558 \\
\hline \multirow[t]{6}{*}{ Figure $1(\mathrm{~d})$} & $0-10 \%$ & $0.642 \pm 0.162$ & $1.238 \pm 0.233$ & $3.266 \pm 0.256$ & 0.703 & $0.290 \pm 0.046$ & 0.428 \\
\hline & $10-20 \%$ & $0.639 \pm 0.177$ & $1.182 \pm 0.202$ & $3.188 \pm 0.233$ & 0.422 & $0.286 \pm 0.040$ & 0.130 \\
\hline & $20-30 \%$ & $0.626 \pm 0.172$ & $1.128 \pm 0.183$ & $3.105 \pm 0.225$ & 1.278 & $0.282 \pm 0.036$ & 0.398 \\
\hline & $30-40 \%$ & $0.617 \pm 0.168$ & $1.080 \pm 0.184$ & $2.892 \pm 0.217$ & 0.475 & $0.275 \pm 0.040$ & 0.111 \\
\hline & $40-60 \%$ & $0.592 \pm 0.176$ & $0.993 \pm 0.193$ & $2.856 \pm 0.206$ & 1.493 & $0.275 \pm 0.035$ & 0.390 \\
\hline & $60-80 \%$ & $8 \pm 0.188$ & $0.870 \pm 0.170$ & $2.477 \pm 0.200$ & 9.734 & $0.258 \pm 0.038$ & 1.112 \\
\hline \multirow{6}{*}{ Figure $1(\mathrm{e})$} & $0-10 \%$ & $0.640 \pm 0.186$ & $1.278 \pm 0.233$ & $3.463 \pm 0.313$ & 0.685 & $0.300 \pm 0.030$ & 0.223 \\
\hline & $10-20 \%$ & $0.635 \pm 0.182$ & $1.182 \pm 0.205$ & $3.448 \pm 0.322$ & 0.427 & $0.308 \pm 0.028$ & 0.322 \\
\hline & $20-30 \%$ & $0.628 \pm 0.173$ & $1.160 \pm 0.212$ & $3.396 \pm 0.302$ & 1.151 & $0.300 \pm 0.032$ & 0.385 \\
\hline & $30-40 \%$ & $0.613 \pm 0.178$ & $1.129 \pm 0.189$ & $3.365 \pm 0.295$ & 0.756 & $0.298 \pm 0.028$ & 0.213 \\
\hline & $40-60 \%$ & $0.608 \pm 0.180$ & $1.098 \pm 0.185$ & $3.242 \pm 0.290$ & 0.398 & $0.285 \pm 0.030$ & 0.242 \\
\hline & $60-80 \%$ & $0.596 \pm 0.177$ & $0.905 \pm 0.190$ & $2.750 \pm 0.293$ & 4.921 & $0.277 \pm 0.027$ & 0.842 \\
\hline
\end{tabular}




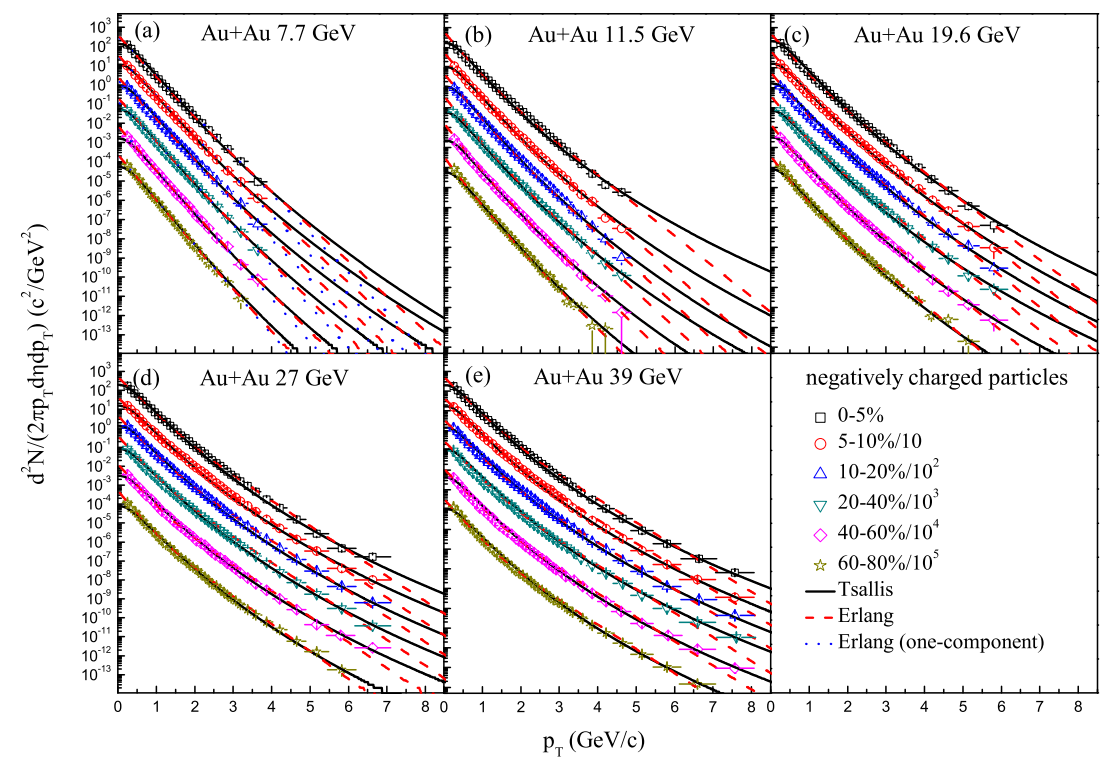

Figure 3. The same as for Fig. 1, but showing the results of negatively charged particles in $|\eta|<0.5$ in different centrality intervals, where the symbols represent the data of the STAR Collaboration [36], and the dashed and solid curves are our results calculated by using the two-component Erlang distribution and the single Tsallis statistics respectively. The dotted curves in Figure 3(a) are an example calculated by using the one-component Erlang distribution.

Table 2. Values of parameters $k_{S 1}, \kappa_{1}, \kappa_{2}$, and $\left\langle p_{t}\right\rangle$, as well as $\chi^{2} /$ dof corresponding to the solid and dashed curves in Fig. 2. In all the cases, for the single Erlang distribution, we have $n=3$ which are not listed in the column.

\begin{tabular}{|c|c|c|c|c|c|c|c|}
\hline \multirow[b]{2}{*}{ Figure } & \multirow[b]{2}{*}{ Type } & \multicolumn{4}{|c|}{ Two-component Schwinger mechanism } & \multicolumn{2}{|c|}{ Single Erlang distribution } \\
\hline & & $k_{S 1}$ & $\kappa_{1}(\mathrm{GeV} / \mathrm{fm})$ & $\kappa_{2}(\mathrm{GeV} / \mathrm{fm})$ & $\chi^{2} / \mathrm{dof}$ & $\left\langle p_{t}\right\rangle(\mathrm{GeV} / c)$ & $\chi^{2} /$ dof \\
\hline \multirow[t]{2}{*}{ Figure 2(a) } & $0-60 \%$ & $0.652 \pm 0.118$ & $1.564 \pm 0.388$ & $2.870 \pm 0.298$ & 0.145 & $0.280 \pm 0.026$ & 0.712 \\
\hline & $0-60 \%$ & $0.652 \pm 0.103$ & $1.580 \pm 0.350$ & $2.912 \pm 0.303$ & 0.270 & $0.282 \pm 0.022$ & 0.828 \\
\hline \multirow{4}{*}{ Figure 2(b) } & $0-10 \%$ & $0.635 \pm 0.115$ & $1.788 \pm 0.373$ & $3.432 \pm 0.305$ & 0.304 & $0.321 \pm 0.031$ & 0.446 \\
\hline & $0-10 \%$ & $0.635 \pm 0.103$ & $1.782 \pm 0.315$ & $3.420 \pm 0.300$ & 0.104 & $0.302 \pm 0.028$ & 0.288 \\
\hline & $10-60 \%$ & $0.632 \pm 0.126$ & $1.753 \pm 0.310$ & $3.213 \pm 0.288$ & 0.209 & $0.293 \pm 0.032$ & 0.291 \\
\hline & $10-60 \%$ & $0.630 \pm 0.118$ & $1.732 \pm 0.272$ & $3.202 \pm 0.282$ & 0.329 & $0.288 \pm 0.030$ & 0.659 \\
\hline \multirow[t]{8}{*}{ Figure 2(c) } & $0-10 \%$ & $0.642 \pm 0.120$ & $1.852 \pm 0.332$ & $3.725 \pm 0.332$ & 0.124 & $0.325 \pm 0.035$ & 0.264 \\
\hline & $0-10 \%$ & $0.635 \pm 0.117$ & $1.786 \pm 0.327$ & $3.693 \pm 0.318$ & 0.134 & $0.315 \pm 0.027$ & 0.286 \\
\hline & $10-20 \%$ & $0.637 \pm 0.122$ & $1.715 \pm 0.303$ & $3.387 \pm 0.302$ & 0.042 & $0.303 \pm 0.030$ & 0.191 \\
\hline & $10-20 \%$ & $0.630 \pm 0.118$ & $1.706 \pm 0.308$ & $3.372 \pm 0.307$ & 0.037 & $0.310 \pm 0.032$ & 0.068 \\
\hline & $20-40 \%$ & $0.627 \pm 0.124$ & $1.658 \pm 0.312$ & $3.243 \pm 0.297$ & 0.109 & $0.306 \pm 0.034$ & 0.100 \\
\hline & $20-40 \%$ & $0.613 \pm 0.113$ & $1.643 \pm 0.298$ & $3.224 \pm 0.290$ & 0.186 & $0.300 \pm 0.032$ & 0.557 \\
\hline & $40-60 \%$ & $0.610 \pm 0.124$ & $1.543 \pm 0.292$ & $3.178 \pm 0.288$ & 0.091 & $0.296 \pm 0.028$ & 0.128 \\
\hline & $40-60 \%$ & $0.602 \pm 0.118$ & $1.482 \pm 0.295$ & $3.076 \pm 0.284$ & 0.121 & $0.290 \pm 0.030$ & 0.295 \\
\hline \multirow{8}{*}{ Figure $2(\mathrm{~d})$} & $0-10 \%$ & $0.657 \pm 0.108$ & $2.420 \pm 0.372$ & $3.878 \pm 0.382$ & 0.049 & $0.343 \pm 0.028$ & 0.215 \\
\hline & $0-10 \%$ & $0.635 \pm 0.102$ & $2.395 \pm 0.398$ & $3.876 \pm 0.400$ & 0.035 & $0.349 \pm 0.035$ & 0.117 \\
\hline & $10-20 \%$ & $0.644 \pm 0.111$ & $2.246 \pm 0.364$ & $3.772 \pm 0.362$ & 0.041 & $0.326 \pm 0.030$ & 0.218 \\
\hline & $10-20 \%$ & $0.630 \pm 0.114$ & $2.356 \pm 0.352$ & $3.770 \pm 0.380$ & 0.054 & $0.332 \pm 0.032$ & 0.236 \\
\hline & $20-40 \%$ & $0.612 \pm 0.103$ & $2.176 \pm 0.357$ & $3.757 \pm 0.337$ & 0.097 & $0.340 \pm 0.038$ & 0.210 \\
\hline & $20-40 \%$ & $0.607 \pm 0.100$ & $2.030 \pm 0.360$ & $3.684 \pm 0.364$ & 0.026 & $0.330 \pm 0.030$ & 0.171 \\
\hline & $40-60 \%$ & $0.598 \pm 0.123$ & $1.843 \pm 0.352$ & $3.472 \pm 0.353$ & 0.133 & $0.302 \pm 0.036$ & 0.317 \\
\hline & $40-60 \%$ & $0.590 \pm 0.102$ & $1.662 \pm 0.346$ & $3.010 \pm 0.230$ & 0.179 & $0.282 \pm 0.025$ & 0.610 \\
\hline \multirow[t]{10}{*}{ Figure 2(e) } & $0-10 \%$ & $0.678 \pm 0.120$ & $2.564 \pm 0.324$ & $4.235 \pm 0.325$ & 0.066 & $0.331 \pm 0.026$ & 0.152 \\
\hline & $0-10 \%$ & $0.668 \pm 0.124$ & $2.478 \pm 0.328$ & $4.192 \pm 0.302$ & 0.128 & $0.335 \pm 0.028$ & 0.087 \\
\hline & $10-20 \%$ & $0.653 \pm 0.115$ & $2.346 \pm 0.336$ & $4.056 \pm 0.316$ & 0.061 & $0.328 \pm 0.032$ & 0.180 \\
\hline & $10-20 \%$ & $0.647 \pm 0.117$ & $2.260 \pm 0.320$ & $3.928 \pm 0.318$ & 0.199 & $0.333 \pm 0.027$ & 0.080 \\
\hline & $20-40 \%$ & $0.640 \pm 0.108$ & $2.232 \pm 0.318$ & $3.937 \pm 0.303$ & 0.220 & $0.330 \pm 0.025$ & 0.108 \\
\hline & $20-40 \%$ & $0.626 \pm 0.117$ & $2.030 \pm 0.322$ & $3.883 \pm 0.322$ & 0.077 & $0.332 \pm 0.027$ & 0.039 \\
\hline & $40-60 \%$ & $0.615 \pm 0.121$ & $1.856 \pm 0.326$ & $3.764 \pm 0.315$ & 0.074 & $0.315 \pm 0.035$ & 0.209 \\
\hline & $40-60 \%$ & $0.602 \pm 0.126$ & $1.802 \pm 0.298$ & $3.650 \pm 0.308$ & 0.047 & $0.318 \pm 0.032$ & 0.159 \\
\hline & $60-80 \%$ & $0.587 \pm 0.127$ & $1.588 \pm 0.292$ & $3.202 \pm 0.313$ & 0.266 & $0.305 \pm 0.025$ & 0.271 \\
\hline & $60-80 \%$ & $0.580 \pm 0.118$ & $1.526 \pm 0.302$ & $3.110 \pm 0.310$ & 0.118 & $0.302 \pm 0.028$ & 0.224 \\
\hline
\end{tabular}


Table 3. Values of parameters $T, q, k_{E 1},\left\langle p_{t}\right\rangle_{1}$, and $\left\langle p_{t}\right\rangle_{2}$, as well as $\chi^{2} /$ dof corresponding to the solid and dashed curves in Fig. 3. In all the cases, for the two-component Erlang distribution, we have $n_{1}=3$ and $n_{2}=2$ which are not listed in the column.

\begin{tabular}{|c|c|c|c|c|c|c|c|c|}
\hline \multirow[b]{2}{*}{ Figure } & \multirow[b]{2}{*}{ Type } & \multicolumn{3}{|c|}{ Single Tsallis statistics } & \multicolumn{4}{|c|}{ Two-component Erlang distribution } \\
\hline & & $T(\mathrm{GeV})$ & $q$ & $\chi^{2} /$ dof & $k_{E 1}$ & $\left\langle p_{t}\right\rangle_{1}(\mathrm{GeV} / c)$ & $\left\langle p_{t}\right\rangle_{2}(\mathrm{GeV} / c)$ & $\chi^{2} /$ dof \\
\hline \multirow{4}{*}{ Figure 3(a) } & $5-10 \%$ & $0.152 \pm 0.012$ & $1.026 \pm 0.007$ & 1.059 & $0.816 \pm 0.021$ & $0.187 \pm 0.017$ & $0.238 \pm 0.026$ & 2.648 \\
\hline & $10-20 \%$ & $0.151 \pm 0.013$ & $1.025 \pm 0.008$ & 1.924 & $0.812 \pm 0.017$ & $0.189 \pm 0.014$ & $0.232 \pm 0.024$ & 2.156 \\
\hline & $40-60 \%$ & $0.150 \pm 0.013$ & $1.020 \pm 0.008$ & 1.487 & $0.807 \pm 0.023$ & $0.180 \pm 0.010$ & $0.220 \pm 0.018$ & 1.585 \\
\hline & $60-80 \%$ & $0.148 \pm 0.010$ & $1.018 \pm 0.008$ & 2.842 & $0.803 \pm 0.020$ & $0.176 \pm 0.012$ & $0.205 \pm 0.020$ & 1.634 \\
\hline \multirow[t]{4}{*}{ Figure $3(\mathrm{~b})$} & $0-5 \%$ & $0.150 \pm 0.012$ & $1.043 \pm 0.006$ & 5.003 & $0.826 \pm 0.024$ & $0.188 \pm 0.016$ & $0.285 \pm 0.019$ & 2.199 \\
\hline & $5-10 \%$ & $0.152 \pm 0.014$ & $1.034 \pm 0.008$ & 3.678 & $0.824 \pm 0.027$ & $0.178 \pm 0.013$ & $0.258 \pm 0.022$ & 1.657 \\
\hline & $40-60 \%$ & $0.150 \pm 0.014$ & $1.030 \pm 0.007$ & 2.983 & $0.786 \pm 0.024$ & $0.168 \pm 0.015$ & $0.234 \pm 0.017$ & 2.460 \\
\hline & $60-80 \%$ & $0.148 \pm 0.008$ & $1.027 \pm 0.008$ & 4.885 & $0.773 \pm 0.023$ & $0.163 \pm 0.013$ & $0.225 \pm 0.016$ & 4.242 \\
\hline \multirow[t]{6}{*}{ Figure 3(c) } & $0-5 \%$ & $0.152 \pm 0.010$ & $1.038 \pm 0.010$ & 4.495 & $0.835 \pm 0.027$ & $0.196 \pm 0.018$ & $0.297 \pm 0.023$ & 1.840 \\
\hline & $5-10 \%$ & \pm 0.012 & $1.035 \pm 0.006$ & 5.609 & $0.826 \pm 0.023$ & $0.187 \pm 0.013$ & $0.288 \pm 0.017$ & 1.180 \\
\hline & $10-20 \%$ & $0.153 \pm 0.010$ & $1.036 \pm 0.007$ & 8.570 & $0.819 \pm 0.025$ & $0.183 \pm 0.016$ & $0.285 \pm 0.019$ & 2.184 \\
\hline & $20-40 \%$ & \pm 0.008 & $1.039 \pm 0.006$ & 3.641 & $0.808 \pm 0.018$ & $0.178 \pm 0.020$ & $0.285 \pm 0.023$ & 3.866 \\
\hline & $40-60 \%$ & \pm 0.013 & $1.037 \pm 0.008$ & 3.354 & $0.795 \pm 0.022$ & $0.173 \pm 0.014$ & $0.280 \pm 0.018$ & 3.457 \\
\hline & $60-80 \%$ & $0.150 \pm 0.011$ & $1.034 \pm 0.006$ & 5.860 & $0.793 \pm 0.024$ & $0.168 \pm 0.017$ & $0.272 \pm 0.020$ & 2.821 \\
\hline Figure 3(d) & $60-80 \%$ & $0.148 \pm 0.012$ & $1.040 \pm 0.007$ & 5.483 & $0.853 \pm 0.027$ & $0.178 \pm 0.013$ & $0.300 \pm 0.022$ & 5.546 \\
\hline \multirow[t]{6}{*}{ Figure 3(e) } & $0-5 \%$ & $0.150 \pm 0.013$ & $1.050 \pm 0.007$ & 3.193 & $0.920 \pm 0.035$ & $0.226 \pm 0.020$ & $0.370 \pm 0.024$ & 6.894 \\
\hline & $5-10 \%$ & $0.152 \pm 0.014$ & $1.048 \pm 0.006$ & 4.982 & $0.913 \pm 0.027$ & $0.223 \pm 0.017$ & $0.368 \pm 0.027$ & 3.643 \\
\hline & $10-20 \%$ & $0.153 \pm 0.012$ & $1.048 \pm 0.007$ & 4.178 & $0.910 \pm 0.032$ & $0.218 \pm 0.015$ & $0.365 \pm 0.022$ & 5.659 \\
\hline & $20-40 \%$ & \pm 0.014 & $1.049 \pm 0.009$ & 9.191 & $0.908 \pm 0.026$ & $0.216 \pm 0.017$ & $0.358 \pm 0.025$ & 9.063 \\
\hline & $40-60 \%$ & $0.151 \pm 0.012$ & $1.049 \pm 0.006$ & 6.714 & $0.902 \pm 0.028$ & $0.210 \pm 0.014$ & $0.356 \pm 0.023$ & 6.465 \\
\hline & $60-80 \%$ & $0.150 \pm 0.014$ & $1.049 \pm 0.008$ & 4.322 & $0.895 \pm 0.025$ & $0.200 \pm 0.015$ & $0.352 \pm 0.024$ & 6.094 \\
\hline
\end{tabular}

To give a comparison, Figure 3 gives the results, $d^{2} N /\left(2 \pi p_{T} d \eta d p_{T}\right)$, of negatively charged particles (hadrons) $\left(h^{-}\right)$produced in mid-pseudorapidity $(|\eta|<0.5)$ in the same collisions with a bit difference in centrality intervals, where $h^{-}$are assumed to produce mostly in soft process and the contribution of hard process to $h^{-}$with high $p_{T}$ is neglected due to small amount. The symbols represent the data of the STAR Collaboration [36], and the dashed and solid curves are our results calculated by using the two-component Erlang distribution and the single Tsallis statistics respectively. The dotted curves in Figure 3(a) are the results of single Erlang distribution, which are given as an example and are not in agreement with the spectra in wide transverse momentum range. The values of $n_{1}$ and $n_{2}$ for all the cases are taken to be 3 and 2 respectively. Other related parameter values related to the two-component Erlang distribution and the single Tsallis statistics are listed in Table 3 with values of $\chi^{2} /$ dof. The chemical potentials related to collision energy [37] in Eq. (5) are taken to be 0.407, 0.304, 0.197, 0.149 , and $0.107 \mathrm{GeV}$ which correspond to $7.7,11.5,19.6,27$, and $39 \mathrm{GeV}$, respectively. For the dotted curves in Figure 3(a) and the centralities from $0-5 \%$ to $60-80 \%$, the values of $\left\langle p_{t}\right\rangle$ in the single Erlang distribution are taken to be $0.212 \pm 0.025,0.210 \pm 0.023,0.209 \pm 0.022,0.203 \pm 0.018,0.194 \pm 0.021$, and $0.186 \pm 0.022 \mathrm{GeV} / c$ and the values of $n$ for all the six cases are taken to be 3, which result in the values of $\chi^{2} /$ dof to be $0.922,2.430$, $1.980,0.930,1.374$, and 1.965, respectively. One can see that the two-component Erlang distribution and the single Tsallis statistics describe approximately the experimental data of $h^{-}$produced in mid-pseudorapidity in $\mathrm{Au}-\mathrm{Au}$ collisions at the RHIC BES in most cases.

To study the change trends of parameters with centrality interval $(C)$ and collision energy $\left(\sqrt{s_{N N}}\right)$, Figure 4 shows the dependences of parameters $k_{S 1}, \kappa_{1}, \kappa_{2}$, and $\left\langle p_{t}\right\rangle$ on centrality at different energies (left panel) and on energy in different centrality intervals (right panel). The different symbols represent the parameter values extracted from Figure 1 and listed in Table 1, where the two-component Schwinger mechanism and single Erlang distribution are used for $\phi$ spectra. The lines are our fitted results, and the values of intercepts, slopes, and $\chi^{2} /$ dof are listed in Table 4. Figure 5 is the same as that for Figure 4, but it shows the parameter values extracted from Figure 2 and listed in Table 2, where the two-component Schwinger mechanism and single Erlang distribution are used for $\Omega^{-}$(solid symbols) and $\bar{\Omega}^{+}$(open symbols) spectra respectively. In particular, the solid and open symbols in Figure 5 are shifted respectively to the left and right sides by a small amount for clearness. The values of intercepts, slopes, and $\chi^{2} /$ dof corresponding to the lines in Figure 5 are listed in Table 5 . Figure 6 displays the dependences of parameters $T, q, k_{E 1},\left\langle p_{t}\right\rangle_{1}$, and $\left\langle p_{t}\right\rangle_{2}$ on centrality at different energies (left panel) and on energy in different centrality intervals (right panel). The different symbols represent the parameter values extracted from Figure 3 and listed in Table 3, where the single Tsallis statistics and two-component Erlang distribution are used for negatively charged particle spectra. The values of intercepts, slopes, and $\chi^{2} /$ dof corresponding to the lines in Figure 6 are listed in Table 6.

One can see from Figures 4-6 that the relative contribution of the first component in the two-component Schwinger mechanism decreases slightly with decrease of centrality, and does not show an obvious change with 
change of collision energy [Figures 4(a), 4(b), 5(a), and 5(b)]. The relative contribution of the first component in the two-component Erlang distribution decreases slightly with decrease of centrality, and increases with increase of collision energy [Figures 6(e) and 6(f)]. The mean transverse momentum contributed by each parton in the Erlang distribution decrease with decrease of centrality [Figures $4(\mathrm{~g}), 5(\mathrm{~g}), 6(\mathrm{~g})$, and $6(\mathrm{i})$ ], and increase with increase of collision energy [Figures 4(h), 5(h),6(h), and 6(j)]. The string tension between partons in the Schwinger mechanism decrease also with decrease of centrality [Figures 4(c), 4(e), 5(c), and 5(e)], and increase also with increase of collision energy [Figures 4(d), 4(f), 5(d), and 5(f)]. The effective temperature of emission source in the Tsallis statistics does not show an obvious dependence on centrality and collision energy [Figure 6(a) and $6(b)]$. The entropy index does not show an obvious change with change of centrality, and increase with increase of collision energy [Figures 6(c) and 6(d)]. A weak softest point in the string tension in $\Omega$ hyperon spectra is observed at $7.7 \mathrm{GeV}$ [Figures 5(e) and 5(g)].

To study the relations between different distributions, as well as their parameters, we could give some further discussions. It should be noted that Figures 1 and 2 devoted to multi-strange hadrons $\phi(s \bar{s})$ mesons and $\Omega(s s s)$ hyperons respectively. Both $\phi(s \bar{s})$ mesons and $\Omega(s s s)$ hyperons are expected to have relatively small hadronic interaction cross-sections. They are also important probes for the search of the QGP phase transition [35]. We have used the two-component Schwinger mechanism and the single Erlang distribution to describe the spectra of multi-strange hadrons which are expected to be produced in hard scattering process. Although the Schwinger mechanism can give the string tension $\kappa$ between partons, the flexible Erlang distribution can describe the violent degree of parton-parton interactions or excitation degree of interacting system (emission source) by the mean transverse momentum $\left\langle p_{t}\right\rangle$ contributed by each parton and the mean transverse momentum $\left\langle p_{T}\right\rangle$ contributed by $n$ partons. From the comparisons between the Schwinger mechanism and Erlang distribution in Figure 1 and Table 1, we can obtain the linear relations between $\kappa$ and $\left\langle p_{T}\right\rangle$ for $\phi$ mesons in Figures $7(\mathrm{a})$ and $7(\mathrm{~b})$ which correspond respectively to different energies (in various centrality intervals) and to different centrality intervals (at various energies). Similarly, the results for $\Omega\left(\Omega^{-}\right.$and $\left.\bar{\Omega}^{+}\right)$hyperons are presented in Figures $7(\mathrm{c})$ and $7(\mathrm{~d})$ based on Figure 2 and Table 2. Different symbols represent different energies and centrality intervals, where $C_{1}$, $C_{2}, \cdots, C_{6}$ for Figures $7(\mathrm{~b})$ and $7(\mathrm{~d})$ represent respectively and successively the centrality intervals mentioned in the right panels of Figures 4 and 5 , and the lines are our fitted results. The values of intercepts, slopes, and $\chi^{2} /$ dof are listed in Table 7 . One can see that $\kappa$ increases with increase of $\left\langle p_{T}\right\rangle$. There is a one-to-one correspondence between the two-component Schwinger mechanism and the single Erlang distribution.

For the spectra of negatively charged hadrons presented in Figure 3, we have used the single Tsallis statistics and the two-component Erlang distribution. Because of the Tsallis statistics resulting in the sum of two or three standard distribution [38], it has the temperature parameter $T$ which describes the excitation degree of interacting system. The mean transverse momentum $\left\langle p_{T}\right\rangle$ obtained from the two-component Erlang distribution also describes the excitation degree of interacting system. From the comparisons between the single Tsallis statistics and the two-component Erlang distribution in Figure 3 and Table 3, we can obtain the linear relations between $T$ and $\left\langle p_{T}\right\rangle$ in Figures $7(\mathrm{e})$ and $7(\mathrm{f})$ which correspond respectively to different collision energies (in various centrality intervals) and to different centrality intervals (at various energies), where $C_{1}, C_{2}, \cdots, C_{6}$ for Figure 7(f) represent successively the centrality intervals mentioned in the right panel of Figure 6 . The values of intercepts, slopes, and $\chi^{2} /$ dof are listed in Table 7 . One can see that $T$ does not show an obvious change with increase of $\left\langle p_{T}\right\rangle$. Instead, Figure 6 shows that $q$ and $\left\langle p_{T}\right\rangle$ increase with increase of $\sqrt{s_{N N}}$, which renders that $q$ increases with increase of $\left\langle p_{T}\right\rangle$. There is a one-to-one correspondence between the single Tsallis statistics and the two-component Erlang distribution. Because the Schwinger mechanism and Tsallis statistics do not describe the same set of experimental data. We do not expect to study the relation between $\kappa$ and $T$. In addition, in the case of considering the two-component functions, we obtain the values of $\kappa, T$, and $\left\langle p_{T}\right\rangle$ discussed above to be respectively an average weighted by the two components.

In our recent works $[39,40]$, we have studied the two-component Schwinger mechanism and the two-component Erlang distribution for $J / \psi$ and $\Upsilon$ productions [39], and Lévy distribution and the multi-component Erlang distribution for identified particles productions [40]. In most cases, different functions (Schwinger and Eralng or Tsallis and Erlang) describe the same set of experimental data (hard or soft particles), which reflects the relations among them. The relations between $\kappa$ and $\left\langle p_{T}\right\rangle$, as well as $T$ and $\left\langle p_{T}\right\rangle$, discussed above are an attempt to study these relations. In particular, the single and two-component Erlang distributions describe the particle spectra in hard and soft processes respectively, which reflects the Erlang distribution being flexible. We think that there are some universal laws existing in the two processes. If the hard process corresponds to a violent collision, the soft process corresponds to a non-violent or a very non-violent collision. Only from the collision itself, both the hard and soft processes obey the same Erlang distribution with different $\left\langle p_{t}\right\rangle$ and parton numbers. We think that the Erlang distribution is one of the universal laws existing in the hard and soft processes.

\section{Conclusions}

We summarize here our main observations and conclusions.

a) The transverse momentum distributions of $\phi$ mesons, $\Omega$ hyperons, and negatively charged particles produced in mid-(pseudo)rapidity in Au-Au collisions with different centrality intervals at RHIC BES energies are analyzed by using the single or two-component Erlang distribution, the two-component Schwinger mechanism, and the single Tsallis statistics. The single or two-component Erlang distribution is approximately in agreement with the experimental data measured by the STAR Collaboration over an energy range from 7.7 to $39 \mathrm{GeV}$. The two-component Schwinger mechanism describes approximately the data of $\phi$ mesons and $\Omega$ hyperons which are 
assumed to produce in hard process. The single Tsallis statistics describes approximately the data of negatively charged particles in which most are assumed to produce in soft process.

b) The mean transverse momentum contributed by each parton in the Erlang distribution which describes $\phi$ mesons, $\Omega$ hypersons, and negatively charged particles, and the string tension between partons in the Schwinger mechanism which describes $\phi$ mesons and $\Omega$ hypersons, decrease with decrease of centrality, and increase with increase of collision energy. The effective temperature of emission source in the Tsallis statistics which describes the negatively charged particles does not show an obvious dependence on centrality and collision energy. The entropy index does not show an obvious change with change of centrality, and increase with increase of collision energy. A weak softest point in the string tension in $\Omega$ hyperon spectra is observed at $7.7 \mathrm{GeV}$.

c) The relative contribution of the first component in the two-component Erlang distribution which describes the negatively charged particles decreases slightly with decrease of centrality, and increases with increase of collision energy. The relative contribution of the first component in the two-component Schwinger mechanism which describes $\phi$ mesons and $\Omega$ hypersons decreases slightly with decrease of centrality, and does not show an obvious change with change of collision energy.

d) In the descriptions of hard process, the string tension in the two-component Schwinger mechanism increases with increase of the mean transverse momentum in the single Erlang distribution. There is a one-to-one correspondence between the two descriptions for hard process. In the descriptions of soft process, the temperature and entropy index in the single Tsallis statistics unchanges approximately or increases with increase of mean transverse momentum in the two-component Erlang distribution. There is also a one-to-one correspondence between the two descriptions for soft process.

\section{Conflict of interests}

The authors declare that there is no conflict of interests regarding the publication of this paper.

\section{Acknowledgments}

This work was supported by the National Natural Science Foundation of China under Grant No. 11575103 and the US DOE under contract DE-FG02-87ER40331.A008. 

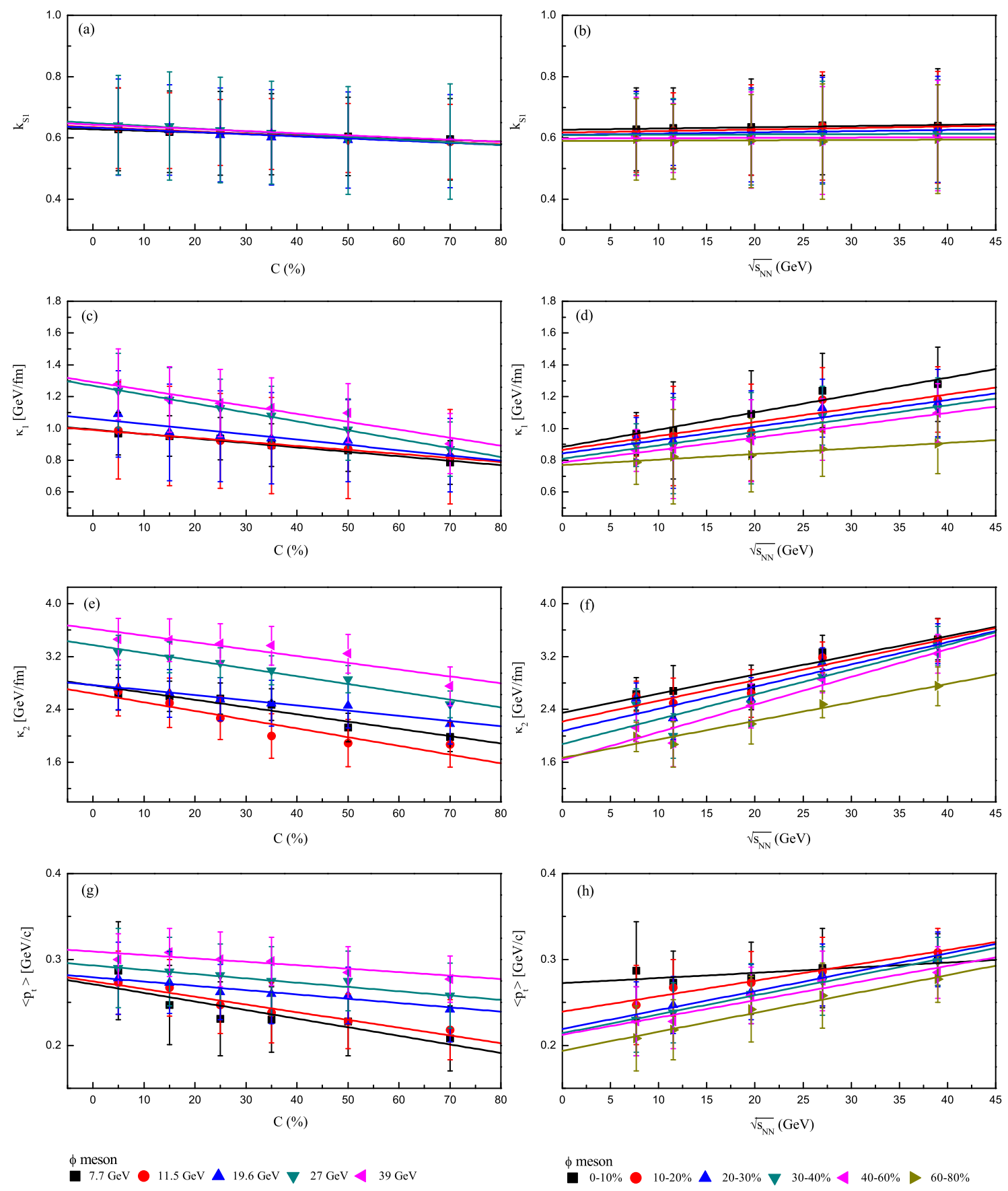

Figure 4. Dependences of parameters (a,b) $k_{S 1},(\mathrm{c}, \mathrm{d}) \kappa_{1},(\mathrm{e}, \mathrm{f}) \kappa_{2}$, and $(\mathrm{g}, \mathrm{h})\left\langle p_{t}\right\rangle$ on centrality at different energies (left panel) and on energy in different centrality intervals (right panel). The different symbols represent the parameter values extracted from Fig. 1 and listed in Table 1, where the two-component Schwinger mechanism and single Erlang distribution are used for $\phi$ spectra. The lines are our fitted results. 
Table 4. Values of intercepts, slopes, and $\chi^{2} /$ dof corresponding to the lines in Fig. 4 which shows dependences of parameters on centrality at different energies (a,c,e,g) and on energy in different centrality intervals (b,d,f,h). The values of parameters are extracted from Fig. 1 and listed in Table 1.

\begin{tabular}{|c|c|c|c|c|c|}
\hline Figure & Relation & Type & Intercept & Slope & $\chi^{2} /$ dof \\
\hline \multirow{5}{*}{ Figure 4(a) } & \multirow{5}{*}{$k_{S 1}-C$} & & Two-compone & Schwinger mechanism & \\
\hline & & $7.7 \mathrm{GeV}$ & $0.628 \pm 0.001$ & $-0.00047 \pm 0.00002$ & 0.001 \\
\hline & & $11.5 \mathrm{GeV}$ & $0.635 \pm 0.001$ & $-0.00067 \pm 0.00002$ & 0.001 \\
\hline & & $19.6 \mathrm{GeV}$ & $0.633 \pm 0.004$ & $-0.00072 \pm 0.00011$ & 0.002 \\
\hline & & $27 \mathrm{GeV}$ & $0.649 \pm 0.004$ & $-0.00094 \pm 0.00010$ & 0.001 \\
\hline \multirow{6}{*}{ Figure 4(b) } & \multirow{6}{*}{$k_{S 1}-\sqrt{s_{N N}}$} & $0-10 \%$ & $\begin{array}{l}0.044 \pm 0.001 \\
0.627 \pm 0.002\end{array}$ & $0.00040 \pm 0.00011$ & $\begin{array}{l}0.001 \\
0.001\end{array}$ \\
\hline & & $10-20 \%$ & $0.618+0.004$ & $0,00054+0.00016$ & 0.001 \\
\hline & & $20-30 \%$ & $0.610 \pm 0.005$ & $0.00044 \pm 0.00020$ & 0.002 \\
\hline & & $30-40 \%$ & $0.610 \pm 0.005$ & $0.00008 \pm 0.00022$ & 0.002 \\
\hline & & $40-60 \%$ & $0.598 \pm 0.007$ & $0.00006 \pm 0.00028$ & 0.003 \\
\hline & & $60-80 \%$ & $0.590 \pm 0.004$ & $0.00006 \pm 0.00016$ & 0.001 \\
\hline \multirow[t]{4}{*}{ Figure 4(c) } & \multirow[t]{4}{*}{$\kappa_{1}-C$} & $7.7 \mathrm{GeV}$ & $0.993 \pm 0.007$ & $-0.00280 \pm 0.00019$ & 0.007 \\
\hline & & $11.5 \mathrm{GeV}$ & $0.990 \pm 0.006$ & $-0.00246 \pm 0.00015$ & 0.001 \\
\hline & & $19.6 \mathrm{GeV}$ & $1.062 \pm 0.024$ & $-0.00329 \pm 0.00059$ & 0.020 \\
\hline & & $39 \mathrm{GeV}$ & $\begin{array}{l}1.209 \\
1.292 \pm 0.026\end{array}$ & $\begin{array}{l}-0.00201 \\
-0.00500+0.00010\end{array}$ & 0.0012 \\
\hline \multirow[t]{6}{*}{ Figure $4(\mathrm{~d})$} & \multirow[t]{6}{*}{$\kappa_{1}-\sqrt{s_{N N}}$} & $0-10 \%$ & $0.883 \pm 0.034$ & $0.01093 \pm 0.00141$ & 0.030 \\
\hline & & $10-20 \%$ & $0.866 \pm 0.050$ & $0.00874 \pm 0.00212$ & 0.079 \\
\hline & & $20-30 \%$ & $0.843 \pm 0.042$ & $0.00844 \pm 0.00175$ & 0.063 \\
\hline & & $30-40 \%$ & $0.810 \pm 0.030$ & $0.00844 \pm 0.00124$ & 0.032 \\
\hline & & $40-60 \%$ & $0.786 \pm 0.012$ & $0.00777 \pm 0.00049$ & 0.007 \\
\hline & & & $0.769 \pm 0.009$ & $0.00354 \pm 0.00037$ & 0.003 \\
\hline \multirow{4}{*}{ Figure $4(\mathrm{e})$} & \multirow{4}{*}{$\kappa_{2}-C$} & $7.7 \mathrm{GeV}$ & $2.640 \pm 0.095$ & $-0.01105 \pm 0.00140$ & 0.127 \\
\hline & & $11.5 \mathrm{GeV}$ & $2.771 \pm 0.030$ & $-0.01316 \pm 0.00238$ & 0.165 \\
\hline & & $19.6 \mathrm{GeV}$ & $3.374 \pm 0.040$ & $-0.00783 \pm 0.00077$ & 0.020 \\
\hline & & $27 \mathrm{GeV}$ & $3.620 \neq 0.085$ & $-0.01180 \pm 0.00101$ & 0.080 \\
\hline \multirow[t]{6}{*}{ Figure 4(f) } & \multirow[t]{6}{*}{$\kappa_{2}-\sqrt{s_{N N}}$} & $0-10 \%$ & $2.351 \pm 0.112$ & $0.02887 \pm 0.00469$ & 0.213 \\
\hline & & $10-20 \%$ & $2.217 \pm 0.132$ & $0.03142 \pm 0.00553$ & 0.326 \\
\hline & & $20-30 \%$ & $2.073 \pm 0.177$ & $0.03357 \pm 0.00743$ & 0.643 \\
\hline & & $30-40 \%$ & $1.876 \pm 0.220$ & $0.03763 \pm 0.00924$ & 0.948 \\
\hline & & $40-60 \%$ & $1.638 \pm 0.140$ & $0.04182 \pm 0.00590$ & 0.391 \\
\hline & & $60-80 \%$ & $1.666 \pm 0.081$ & $0.02797 \pm 0.00339$ & 0.140 \\
\hline \multirow{5}{*}{ Figure 4(g) } & \multirow{5}{*}{$\left\langle p_{t}\right\rangle-C$} & & Single 1 & lang distribution in Fig. & 1 \\
\hline & & $7.7 \mathrm{GeV}$ & $0.271 \pm 0.010$ & $-0.00098 \pm 0.00025$ & 0.095 \\
\hline & & $11.5 \mathrm{GeV}$ & $0.275 \pm 0.004$ & $-0.00088 \pm 0.00010$ & 0.029 \\
\hline & & $19.6 \mathrm{GeV}$ & $0.279 \pm 0.002$ & $-0.00051 \pm 0.00006$ & 0.011 \\
\hline & & $27 \mathrm{GeV}$ & $0.293 \pm 0.002$ & $-0.00046 \pm 0.00005$ & 0.007 \\
\hline \multirow{6}{*}{ Figure $4(\mathrm{~h})$} & \multirow{6}{*}{$\left\langle p_{t}\right\rangle-\sqrt{s_{N N}}$} & $0-10 \%$ & $0.272 \pm 0.007$ & $0.00063 \pm 0.00028$ & 0.032 \\
\hline & & $10-20 \%$ & $0.239 \pm 0.004$ & $0.00177 \pm 0.00019$ & 0.023 \\
\hline & & $20-30 \%$ & $0.219 \pm 0.004$ & $0.00216 \pm 0.00016$ & 0.016 \\
\hline & & $30-40 \%$ & $0.214 \pm 0.002$ & $0.00220 \pm 0.00008$ & 0.005 \\
\hline & & $40-60 \%$ & $0.212 \pm 0.007$ & $0.00202 \pm 0.00028$ & 0.062 \\
\hline & & & 004 & $0.00223 \pm 0.00016$ & 0.018 \\
\hline
\end{tabular}



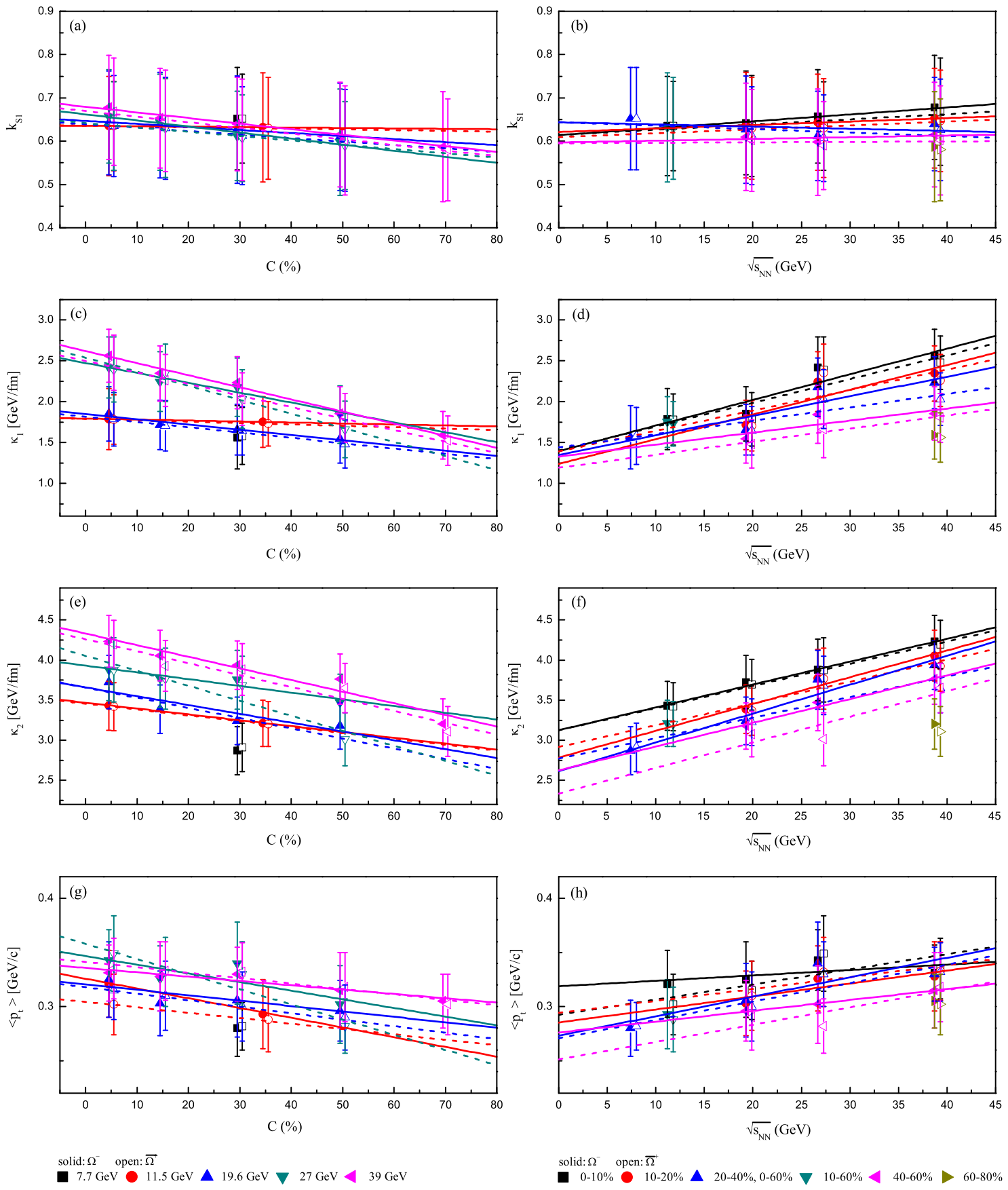

Figure 5. As for Fig. 4, but showing the parameter values extracted from Fig. 2 and listed in Table 2 , where the two-component Schwinger mechanism and single Erlang distribution are used for $\Omega^{-}$(solid symbols) and $\bar{\Omega}^{+}$(open symbols) spectra respectively. The solid and open symbols are shifted respectively to the left and right sides by a small amount for clearness. 
Table 5. Values of intercepts, slopes, and $\chi^{2} /$ dof corresponding to the lines in Fig. 5 which shows dependences of parameters on centrality at different energies (a,c,e,g) and on energy in different centrality intervals (b,d,f,h). The values of parameters are extracted from Fig. 2 and listed in Table 2.

\begin{tabular}{|c|c|c|c|c|c|}
\hline Figure & Relation & Type & Intercept & Slope & $\chi^{2} /$ dof \\
\hline \multirow{9}{*}{ Figure 5(a) } & \multirow{9}{*}{$k_{S 1}-C$} & & Two-componen & Schwinger mechanism & in Fig. 2 \\
\hline & & $11.5 \mathrm{GeV} \Omega^{-}$ & $0.636 \pm 0.000$ & $-0.00009 \pm 0.00000$ & 0.001 \\
\hline & & $11.5 \mathrm{GeV} \bar{\Omega}^{+}$ & $0.636 \pm 0.000$ & $-0.00017 \pm 0.00000$ & 0.001 \\
\hline & & $19.6 \mathrm{GeV} \Omega^{-}$ & $0.647 \pm 0.001$ & $-0.00072 \pm 0.00004$ & 0.001 \\
\hline & & $19.6 \mathrm{GeV} \bar{\Omega}^{+}$ & $0.639 \pm 0.002$ & $-0.00077 \pm 0.00007$ & 0.001 \\
\hline & & $27 \mathrm{GeV} \Omega^{-}$ & $0.662 \pm 0.005$ & $-0.00136 \pm 0.00017$ & 0.005 \\
\hline & & $27 \mathrm{GeV} \bar{\Omega}^{+}$ & $0.642 \pm 0.003$ & $-0.00106 \pm 0.00009$ & 0.001 \\
\hline & & $39 \mathrm{GeV} \Omega^{-}$ & $0.680 \pm 0.003$ & $-0.00132 \pm 0.00008$ & 0.002 \\
\hline & & $39 \mathrm{GeV} \bar{\Omega}^{+}$ & $0.670 \pm 0.003$ & $-0.00132 \pm 0.00007$ & 0.001 \\
\hline \multirow[t]{8}{*}{ Figure 5(b) } & \multirow{8}{*}{$k_{S 1}-\sqrt{s_{N N}}$} & $0-10 \% \Omega^{-}$ & $0.614 \pm 0.003$ & $0.00161 \pm 0.00013$ & 0.001 \\
\hline & & $0-10 \% \bar{\Omega}^{+}$ & $0.614 \pm 0.012$ & $0.00119 \pm 0.00044$ & 0.011 \\
\hline & & $10-20 \% \Omega^{-}$ & $0.621 \pm 0.001$ & $0.00082 \pm 0.00004$ & 0.001 \\
\hline & & $10-20 \% \bar{\Omega}^{+}$ & $0.609 \pm 0.008$ & $0.00093 \pm 0.00027$ & 0.002 \\
\hline & & $20-40 \% \Omega^{-}$ & $0.644 \pm 0.018$ & $-0.00047 \pm 0.00071$ & 0.033 \\
\hline & & $20-40 \% \bar{\Omega}^{+}$ & $0.644 \pm 0.019$ & $-0.00083 \pm 0.00074$ & 0.034 \\
\hline & & $40-60 \% \Omega^{-}$ & $0.597 \pm 0.017$ & $0.00037 \pm 0.00057$ & 0.008 \\
\hline & & $40-60 \% \bar{\Omega}^{+}$ & $0.595 \pm 0.015$ & $0.00010 \pm 0.00050$ & 0.008 \\
\hline \multirow{8}{*}{ Figure $5(\mathrm{c})$} & \multirow{8}{*}{$\kappa_{1}-C$} & $11.5 \mathrm{GeV} \Omega^{-}$ & $1.794 \pm 0.000$ & $-0.00117 \pm 0.00000$ & 0.001 \\
\hline & & $11.5 \mathrm{GeV} \bar{\Omega}^{+}$ & $1.790 \pm 0.000$ & $-0.00167 \pm 0.00000$ & 0.001 \\
\hline & & $19.6 \mathrm{GeV} \Omega^{-}$ & $1.851 \pm 0.027$ & $-0.00637 \pm 0.00090$ & 0.014 \\
\hline & & $19.6 \mathrm{GeV} \bar{\Omega}^{+}$ & $1.818 \pm 0.014$ & $-0.00653 \pm 0.00046$ & 0.004 \\
\hline & & $27 \mathrm{GeV} \Omega^{-}$ & $2.474 \pm 0.043$ & $-0.01209 \pm 0.00144$ & 0.028 \\
\hline & & $27 \mathrm{GeV} \bar{\Omega}^{+}$ & $2.540 \pm 0.050$ & $-0.01718 \pm 0.00164$ & 0.034 \\
\hline & & $39 \mathrm{GeV}^{-}$ & $2.620 \pm 0.032$ & $-0.01480 \pm 0.00078$ & 0.021 \\
\hline & & $39 \mathrm{GeV}^{+}$ & $2.500 \pm 0.029$ & $-0.01414 \pm 0.00071$ & 0.017 \\
\hline \multirow[t]{8}{*}{ Figure 5(d) } & \multirow{8}{*}{$\kappa_{1}-\sqrt{s_{N N}}$} & $0-10 \% \Omega^{-}$ & $1.394 \pm 0.185$ & $0.03140 \pm 0.00702$ & 0.245 \\
\hline & & $0-10 \% \bar{\Omega}^{+}$ & $1.404 \pm 0.216$ & $0.02907 \pm 0.00823$ & 0.326 \\
\hline & & $10-20 \% \Omega^{-}$ & $1.240 \pm 0.355$ & $0.03020 \pm 0.01200$ & 0.469 \\
\hline & & $10-20 \% \bar{\Omega}^{+}$ & $1.393 \pm 0.537$ & $0.02505 \pm 0.01812$ & 1.115 \\
\hline & & $20-40 \% \Omega^{-}$ & $1.350 \pm 0.164$ & $0.02392 \pm 0.00631$ & 0.276 \\
\hline & & $20-40 \% \bar{\Omega}^{+}$ & $1.439 \pm 0.129$ & $0.01638 \pm 0.00497$ & 0.174 \\
\hline & & $40-60 \% \Omega^{-}$ & $1.328 \pm 0.221$ & $0.01470 \pm 0.00746$ & 0.194 \\
\hline & & $40-60 \% \bar{\Omega}^{+}$ & $1.191 \pm 0.071$ & $0.01603 \pm 0.00240$ & 0.021 \\
\hline \multirow[t]{8}{*}{ Figure 5(e) } & \multirow{8}{*}{$\kappa_{2}-C$} & $11.5 \mathrm{GeV} \Omega^{-}$ & $3.468 \pm 0.000$ & $-0.00730 \pm 0.00000$ & 0.001 \\
\hline & & $11.5 \mathrm{GeV} \bar{\Omega}^{+}$ & $3.456 \pm 0.000$ & $-0.00727 \pm 0.00000$ & 0.001 \\
\hline & & $19.6 \mathrm{GeV} \Omega^{-}$ & $3.659 \pm 0.027$ & $-0.01105 \pm 0.00333$ & 0.200 \\
\hline & & $19.6 \mathrm{GeV} \bar{\Omega}^{+}$ & $3.658 \pm 0.014$ & $-0.01266 \pm 0.00266$ & 0.130 \\
\hline & & $27 \mathrm{GeV} \Omega^{-}$ & $3.930 \pm 0.043$ & $-0.00843 \pm 0.00160$ & 0.033 \\
\hline & & $27 \mathrm{GeV} \bar{\Omega}^{+}$ & $4.054 \pm 0.012$ & $-0.01874 \pm 0.00401$ & 0.214 \\
\hline & & $39 \mathrm{GeV} \Omega^{-}$ & $4.331 \pm 0.082$ & $-0.01449 \pm 0.00198$ & 0.147 \\
\hline & & $39 \mathrm{GeV} \bar{\Omega}^{+}$ & $4.260 \pm 0.085$ & $-0.01494 \pm 0.00204$ & 0.158 \\
\hline \multirow[t]{8}{*}{ Figure 5(f) } & \multirow[t]{8}{*}{$\kappa_{2}-\sqrt{s_{N N}}$} & $0-10 \% \Omega^{-}$ & $3.125 \pm 0.037$ & $0.02851 \pm 0.00140$ & 0.001 \\
\hline & & $0-10 \% \bar{\Omega}^{+}$ & $3.123 \pm 0.028$ & $0.02770 \pm 0.00105$ & 0.007 \\
\hline & & $10-20 \% \Omega^{-}$ & $2.784 \pm 0.159$ & $0.03345 \pm 0.00536$ & 0.096 \\
\hline & & $10-20 \% \bar{\Omega}^{+}$ & $2.915 \pm 0.228$ & $0.02717 \pm 0.00768$ & 0.184 \\
\hline & & $20-40 \% \Omega^{-}$ & $2.613 \pm 0.135$ & $0.03600 \pm 0.00521$ & 0.179 \\
\hline & & $20-40 \% \bar{\Omega}^{+}$ & $2.770 \pm 0.173$ & $0.02560 \pm 0.00667$ & 0.292 \\
\hline & & $40-60 \% \Omega^{-}$ & $2.626 \pm 0.086$ & $0.02964 \pm 0.00291$ & 0.030 \\
\hline & & $40-60 \% \bar{\Omega}^{+}$ & $2.336 \pm 0.349$ & $0.03187 \pm 0.01177$ & 0.537 \\
\hline \multirow{9}{*}{ Figure 5(g) } & & & Single Er & ang distribution in Fig. & 2 \\
\hline & $\left\langle p_{t}\right\rangle-C$ & $11.5 \mathrm{GeV} \Omega^{-}$ & $0.326 \pm 0.000$ & $-0.00093 \pm 0.00000$ & 0.001 \\
\hline & & $11.5 \mathrm{GeV} \bar{\Omega}^{+}$ & $0.304 \pm 0.000$ & $-0.00047 \pm 0.00000$ & 0.001 \\
\hline & & $19.6 \mathrm{GeV} \Omega^{-}$ & $0.321 \pm 0.006$ & $-0.00052 \pm 0.00021$ & 0.075 \\
\hline & & $19.6 \mathrm{GeV} \bar{\Omega}^{+}$ & $0.318 \pm 0.001$ & $-0.00056 \pm 0.00002$ & 0.001 \\
\hline & & $27 \mathrm{GeV} \Omega^{-}$ & $0.346 \pm 0.010$ & $-0.00076 \pm 0.00034$ & 0.155 \\
\hline & & $27 \mathrm{GeV} \bar{\Omega}^{+}$ & $0.358 \pm 0.008$ & $-0.00139 \pm 0.00027$ & 0.153 \\
\hline & & $39 \mathrm{GeV} \Omega^{-}$ & $0.336 \pm 0.003$ & $-0.00041 \pm 0.00007$ & 0.030 \\
\hline & & $39 \mathrm{GeV} \bar{\Omega}^{+}$ & $0.341 \pm 0.003$ & $-0.00051 \pm 0.00008$ & 0.030 \\
\hline Figure $5(\mathrm{~h})$ & $\left\langle p_{t}\right\rangle-\sqrt{s_{N N}}$ & $0-10 \% \Omega^{-}$ & $0.319 \pm 0.010$ & $0.00046 \pm 0.00040$ & 0.121 \\
\hline & & $0-10 \% \bar{\Omega}^{+}$ & $0.292 \pm 0.018$ & $0.00135 \pm 0.00067$ & 0.264 \\
\hline & & $10-20 \% \Omega^{-}$ & $0.285 \pm 0.016$ & $0.00118 \pm 0.00056$ & 0.130 \\
\hline & & $10-20 \% \bar{\Omega}^{+}$ & $0.294 \pm 0.016$ & $0.00108 \pm 0.00055$ & 0.116 \\
\hline & & $20-40 \% \Omega^{-}$ & $0.273 \pm 0.016$ & $0.00175 \pm 0.00060$ & 0.271 \\
\hline & & $20-40 \% \bar{\Omega}^{+}$ & $0.271 \pm 0.010$ & $0.00173 \pm 0.00038$ & 0.129 \\
\hline & & $40-60 \% \Omega^{-}$ & $0.276 \pm 0.002$ & $0.00100 \pm 0.00005$ & 0.001 \\
\hline & & $40-60 \% \bar{\Omega}^{+}$ & $0.251 \pm 0.023$ & $0.00159 \pm 0.00077$ & 0.324 \\
\hline
\end{tabular}


Table 6. Values of intercepts, slopes, and $\chi^{2} /$ dof corresponding to the lines in Fig. 6 which shows dependences of parameters on centrality at different energies (a,c,e,g,i) and on energy in different centrality intervals $(b, d, f, h, j)$. The values of parameters are extracted from Fig. 3 and listed in Table 3.

\begin{tabular}{|c|c|c|c|c|c|}
\hline Figure & Relation & Type & Intercept & Slope & $\chi^{2} /$ dof \\
\hline \multirow{6}{*}{ Figure 6(a) } & \multirow{4}{*}{$T-C$} & & \multicolumn{3}{|c|}{ Single Tsallis statistics in Fig. 3} \\
\hline & & $7.7 \mathrm{GeV}$ & $0.152 \pm 0.001$ & $-0.00005 \pm 0.00001$ & 0.001 \\
\hline & & $11.5 \mathrm{GeV}$ & $0.151 \pm 0.001$ & $-0.00004 \pm 0.00001$ & 0.006 \\
\hline & & $19.6 \mathrm{GeV}$ & $0.154 \pm 0.001$ & $-0.00006 \pm 0.00001$ & 0.021 \\
\hline & & $27 \mathrm{GeV}$ & $0.153 \pm 0.001$ & $0.00003 \pm 0.00001$ & 0.005 \\
\hline & \multirow{7}{*}{$T-\sqrt{s_{N N}}$} & $39 \mathrm{GeV}$ & $0.152 \pm 0.001$ & $-0.00002 \pm 0.00001$ & 0.012 \\
\hline \multirow{6}{*}{ Figure $6(\mathrm{~b})$} & & $0-5 \%$ & $0.152 \pm 0.001$ & $-0.00003 \pm 0.00004$ & 0.010 \\
\hline & & $5-10 \%$ & $0.153 \pm 0.002$ & $0.00001 \pm 0.00007$ & 0.027 \\
\hline & & $10-20 \%$ & $0.150 \pm 0.001$ & $0.00010 \pm 0.00004$ & 0.014 \\
\hline & & $20-40 \%$ & $0.150 \pm 0.001$ & $0.00010 \pm 0.00006$ & 0.019 \\
\hline & & $40-60 \%$ & $0.150 \pm 0.002$ & $0.00007 \pm 0.00007$ & 0.028 \\
\hline & & $60-80 \%$ & $0.148 \pm 0.002$ & $0.00012 \pm 0.00010$ & 0.055 \\
\hline \multirow[t]{5}{*}{ Figure 6(c) } & \multirow{5}{*}{$q-C$} & $7.7 \mathrm{GeV}$ & $1.026 \pm 0.001$ & $-0.00011 \pm 0.00002$ & 0.019 \\
\hline & & $11.5 \mathrm{GeV}$ & $1.038 \pm 0.001$ & $-0.00017 \pm 0.00006$ & 0.322 \\
\hline & & $19.6 \mathrm{GeV}$ & $1.037 \pm 0.001$ & $-0.00002 \pm 0.00003$ & 0.098 \\
\hline & & $27 \mathrm{GeV}$ & $1.043 \pm 0.001$ & $-0.00003 \pm 0.00001$ & 0.012 \\
\hline & & $39 \mathrm{GeV}$ & $1.049 \pm 0.001$ & $0.00001 \pm 0.00001$ & 0.015 \\
\hline \multirow[t]{6}{*}{ Figure $6(d)$} & \multirow{6}{*}{$q-\sqrt{s_{N N}}$} & $0-5 \%$ & $1.027 \pm 0.006$ & $0.00061 \pm 0.00024$ & 1.079 \\
\hline & & $5-10 \%$ & $1.024 \pm 0.002$ & $0.00065 \pm 0.00009$ & 0.133 \\
\hline & & $10-20 \%$ & $1.022 \pm 0.001$ & $0.00071 \pm 0.00006$ & 0.053 \\
\hline & & $20-40 \%$ & $1.021 \pm 0.003$ & $0.00076 \pm 0.00014$ & 0.458 \\
\hline & & $40-60 \%$ & $1.018 \pm 0.003$ & $0.00086 \pm 0.00012$ & 0.131 \\
\hline & & $60-80 \%$ & $1.014 \pm 0.002$ & $0.00093 \pm 0.00009$ & 0.120 \\
\hline \multirow{6}{*}{ Figure 6(e) } & \multirow{6}{*}{$k_{E 1}-C$} & & Two-componen & Erlang distribution in & 1 Fig. 3 \\
\hline & & $7.7 \mathrm{GeV}$ & $0.818 \pm 0.001$ & $-0.00023 \pm 0.00004$ & 0.017 \\
\hline & & $11.5 \mathrm{GeV}$ & $0.827 \pm 0.003$ & $-0.00082 \pm 0.00007$ & 0.040 \\
\hline & & $19.6 \mathrm{GeV}$ & $0.831 \pm 0.003$ & $-0.00062 \pm 0.00008$ & 0.053 \\
\hline & & $27 \mathrm{GeV}$ & $0.873 \pm 0.001$ & $-0.00029 \pm 0.00003$ & 0.005 \\
\hline & & $39 \mathrm{GeV}$ & $0.917 \pm 0.001$ & $-0.00032 \pm 0.00004$ & 0.005 \\
\hline \multirow[t]{6}{*}{ Figure $6(\mathrm{f})$} & \multirow[t]{6}{*}{$k_{E 1}-\sqrt{s_{N N}}$} & $0-5 \%$ & $0.787 \pm 0.009$ & $0.00325 \pm 0.00038$ & 0.128 \\
\hline & & $5-10 \%$ & $0.784 \pm 0.011$ & $0.00316 \pm 0.00045$ & 0.315 \\
\hline & & $10-20 \%$ & $0.777 \pm 0.012$ & $0.00324 \pm 0.00050$ & 0.383 \\
\hline & & $20-40 \%$ & $0.762 \pm 0.016$ & $0.00354 \pm 0.00067$ & 1.138 \\
\hline & & $40-60 \%$ & $0.755 \pm 0.020$ & $0.00356 \pm 0.00082$ & 1.094 \\
\hline & & $60-80 \%$ & $0.784 \pm 0.020$ & $0.00359 \pm 0.00084$ & 1.218 \\
\hline \multirow[t]{4}{*}{ Figure $6(\mathrm{~g})$} & \multirow{4}{*}{$\left\langle p_{t}\right\rangle_{1}-C$} & $7.7 \mathrm{GeV}$ & $0.190 \pm 0.001$ & $-0.00020 \pm 0.00002$ & 0.009 \\
\hline & & $11.5 \mathrm{GeV}$ & $0.184 \pm 0.002$ & $-0.00031 \pm 0.00005$ & 0.049 \\
\hline & & $19.6 \mathrm{GeV}$ & $0.191 \pm 0.002$ & $-0.00036 \pm 0.00005$ & 0.044 \\
\hline & & $\begin{array}{l}27 \mathrm{GeV} \\
39 \mathrm{GeV}\end{array}$ & $\begin{array}{l}0.210 \pm 0.002 \\
0.226 \pm 0.001\end{array}$ & $\begin{array}{l}-0.00049 \pm 0.00005 \\
-0.00035 \pm 0.00003\end{array}$ & 0.014 \\
\hline \multirow{6}{*}{ Figure 6(h) } & \multirow{6}{*}{$\left\langle p_{t}\right\rangle_{1}-\sqrt{s_{N N}}$} & $0-5 \%$ & $0.175 \pm 0.003$ & $0.00129 \pm 0.00014$ & 0.064 \\
\hline & & $5-10 \%$ & $0.168 \pm 0.006$ & $0.00135 \pm 0.00026$ & 0.263 \\
\hline & & $10-20 \%$ & $0.170 \pm 0.007$ & $0.00112 \pm 0.00030$ & 0.349 \\
\hline & & $20-40 \%$ & $0.163 \pm 0.008$ & $0.00119 \pm 0.00032$ & 0.383 \\
\hline & & $40-60 \%$ & $0.160 \pm 0.008$ & $0.00110 \pm 0.00034$ & 0.706 \\
\hline & & 60 & \pm 0.008 & $0.00092 \pm 0.00033$ & 0.517 \\
\hline \multirow{5}{*}{ Figure 6(i) } & $\left\langle p_{t}\right\rangle_{2}-C$ & $7.7 \mathrm{GeV}$ & $0.241 \pm 0.001$ & $-0.00049 \pm 0.00004$ & 0.016 \\
\hline & & $11.5 \mathrm{GeV}$ & $0.369 \pm 0.007$ & $-0.00069 \pm 0.00018$ & 0.393 \\
\hline & & $19.6 \mathrm{GeV}$ & $0.293 \pm 0.002$ & $-0.00029 \pm 0.00005$ & 0.029 \\
\hline & & $27 \mathrm{GeV}$ & $0.330 \pm 0.003$ & $-0.00035 \pm 0.00007$ & 0.041 \\
\hline & & $39 \mathrm{GeV}$ & $0.369 \pm 0.001$ & $-0.00026 \pm 0.00003$ & 0.006 \\
\hline Figure 6(j) & $\left\langle p_{t}\right\rangle_{2}-\sqrt{s_{N N}}$ & $0-5 \%$ & $0.225 \pm 0.010$ & $0.00377 \pm 0.00044$ & 0.376 \\
\hline & & $5-10 \%$ & $0.208 \pm 0.003$ & $0.00416 \pm 0.00014$ & 0.029 \\
\hline & & $10-20 \%$ & $0.199 \pm 0.005$ & $0.00438 \pm 0.00019$ & 0.059 \\
\hline & & $20-40 \%$ & $0.196 \pm 0.006$ & $0.00432 \pm 0.00024$ & 0.082 \\
\hline & & $40-60 \%$ & $0.187 \pm 0.007$ & $0.00452 \pm 0.00030$ & 0.156 \\
\hline & & $60-80 \%$ & $0.173 \pm 0.004$ & $0.00468 \pm 0.00018$ & 0.069 \\
\hline
\end{tabular}



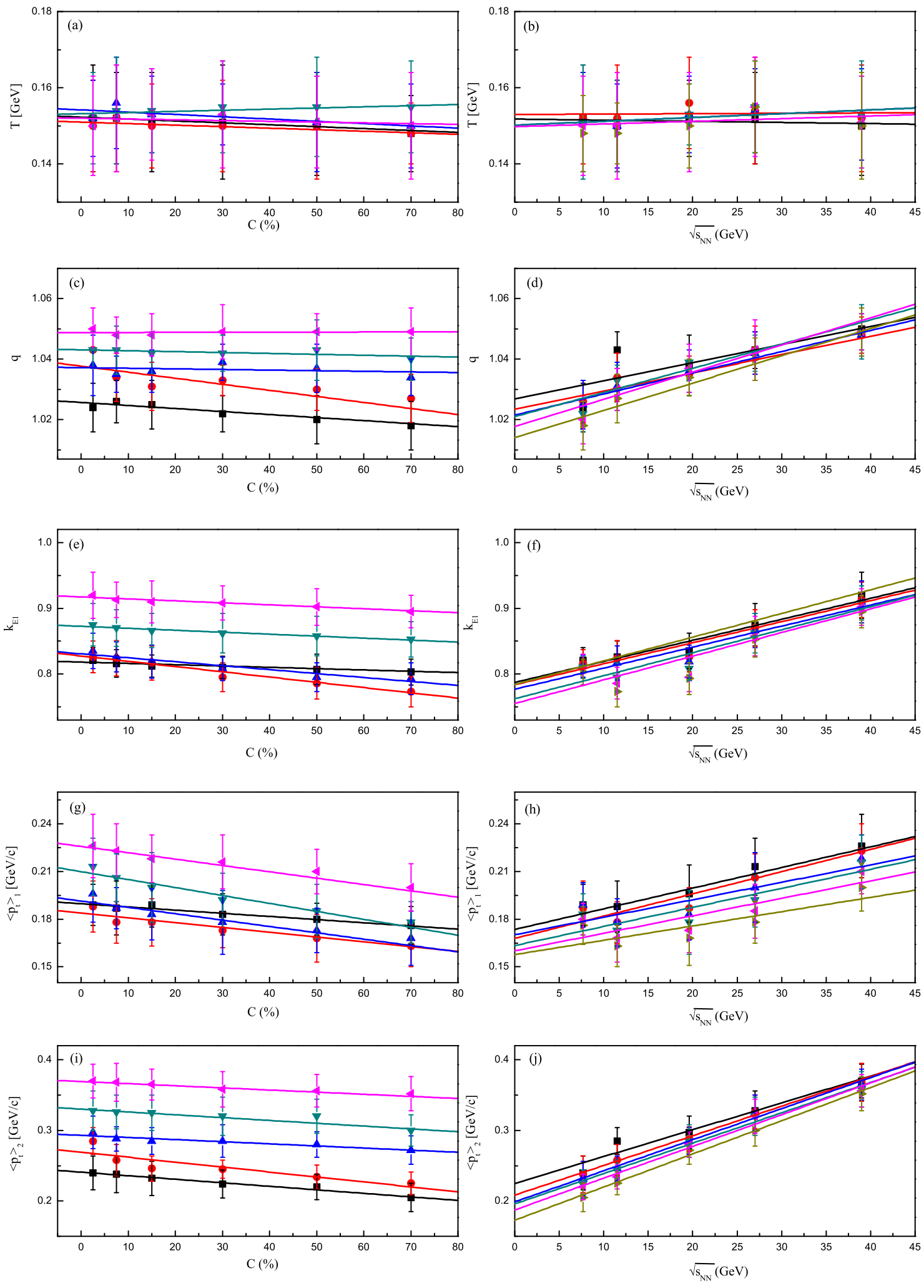

negatively charged particles
$7.7 \mathrm{GeV} 11.5 \mathrm{GeV} \Delta 19.6 \mathrm{GeV} \nabla 27 \mathrm{GeV}<39 \mathrm{GeV}$

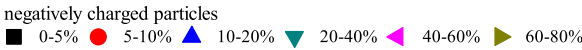

Figure 6. Dependences of parameters (a,b) $T$, (c,d) $q$, (e,f) $k_{E 1}$, (g,h) $\left\langle p_{t}\right\rangle_{1}$, and (i,j) $\left\langle p_{t}\right\rangle_{2}$ on centrality at different energies (left panel) and on energy in different centrality intervals (right panel). The different symbols represent the parameter values extracted from Fig. 3 and listed in Table 3, where the single Tsallis statistics and two-component Erlang distribution are used for negatively charged particle spectra. The lines are our fitted results. 

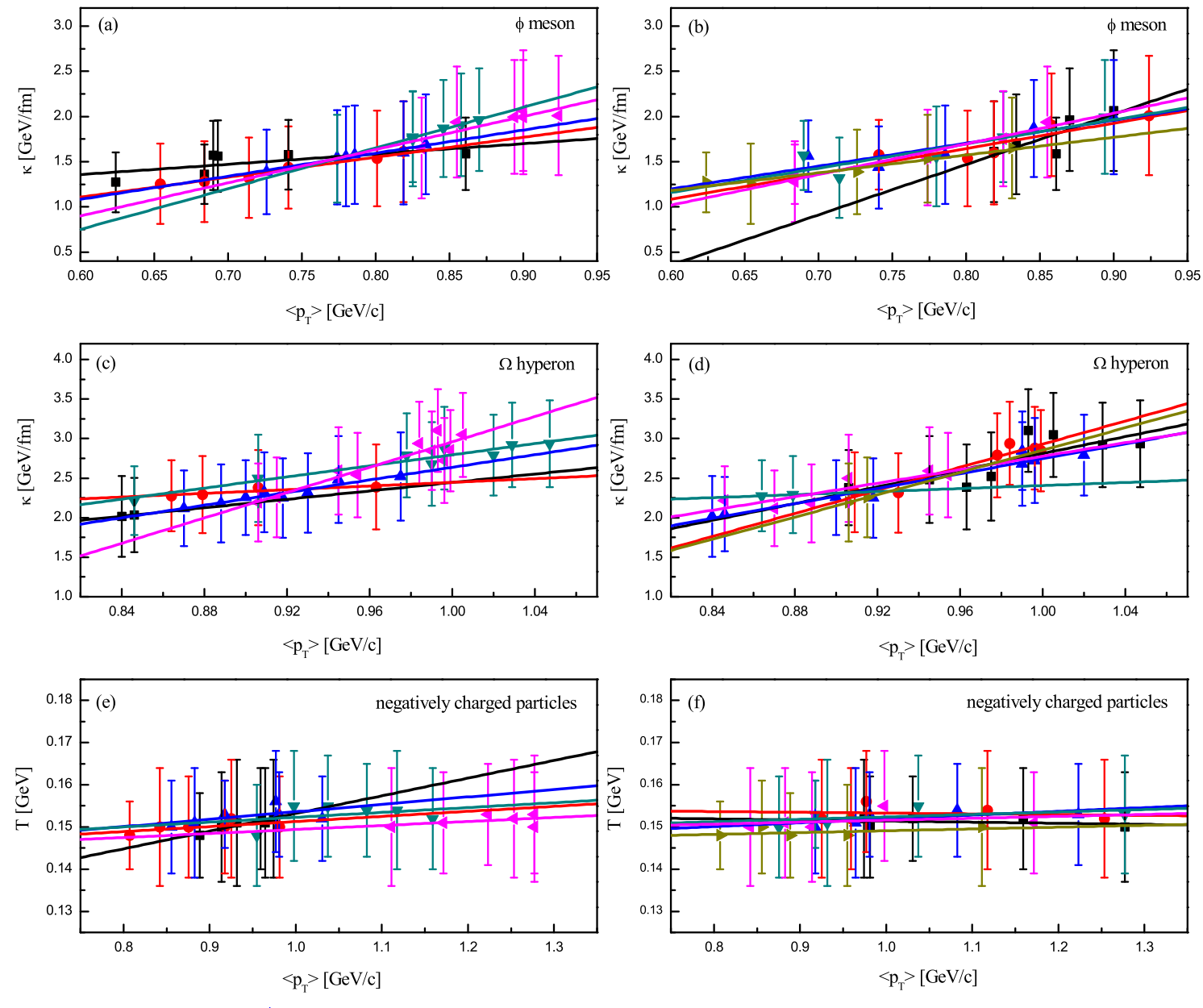

- $7.7 \mathrm{GeV} \bigcirc 11.5 \mathrm{GeV} \Delta 19.6 \mathrm{GeV} \nabla 27 \mathrm{GeV}<39 \mathrm{GeV}$

- $\mathrm{C}_{1} \odot \mathrm{C}_{2} \boldsymbol{\Delta} \mathrm{C}_{3} \nabla \mathrm{C}_{4} \triangleleft \mathrm{C}_{5}>\mathrm{C}_{6}$

Figure 7. Relations between different parameters, (a,b) $\kappa$ and $\left\langle p_{T}\right\rangle$ for $\phi$ mesons, (c,d) $\kappa$ and $\left\langle p_{T}\right\rangle$ for $\Omega$ hyperons, and (e,f) $T$ and $\left\langle p_{T}\right\rangle$ for negatively charged particles. The different symbols represent the parameter values at different energies (in various centrality intervals) (left panel) and in different centrality intervals (at various energies) (right panel), where $C_{1}, C_{2}, \cdots, C_{6}$ for Figures 7 (b), 7(d), and 7 (f) represent the centrality intervals mentioned in the right panels of Figures 4, 5, and 6, respectively. In some cases the parameter values are obtained by an average weighted by two components which are extracted from Figs. 1-3 and listed in Tables 1-3. The lines are our fitted results. 
Table 7. Values of intercepts, slopes, and $\chi^{2} /$ dof corresponding to the lines in Fig. 7 which shows the relations between different parameters.

\begin{tabular}{|c|c|c|c|c|c|}
\hline$\overline{\overline{\text { Figure }}}$ & Relation & Type & Intercept & Slope & $\overline{\chi^{2} / \mathrm{dof}}$ \\
\hline \multirow[t]{5}{*}{ Figure $7(\mathrm{a})$} & $\kappa-\left\langle p_{T}\right\rangle$ & $7.7 \mathrm{GeV}$ & $0.674 \pm 0.406$ & $1.139 \pm 0.564$ & 0.098 \\
\hline & & $11.5 \mathrm{GeV}$ & $-0.218 \pm 0.133$ & $2.208 \pm 0.180$ & 0.004 \\
\hline & & $19.6 \mathrm{GeV}$ & $-0.448 \pm 0.239$ & $2.553 \pm 0.304$ & 0.003 \\
\hline & & $27 \mathrm{GeV}$ & $-1.951 \pm 0.092$ & $4.502 \pm 0.110$ & 0.001 \\
\hline & & $39 \mathrm{GeV}$ & $-1.305 \pm 0.906$ & $3.672 \pm 0.024$ & 0.021 \\
\hline \multirow[t]{6}{*}{ Figure $7(b)$} & $\kappa-\left\langle p_{T}\right\rangle$ & $0-10 \%$ & $-2.984 \pm 1.712$ & $5.565 \pm 1.997$ & 0.117 \\
\hline & & $10-20 \%$ & $-0.596 \pm 0.628$ & $2.802 \pm 0.775$ & 0.057 \\
\hline & & $20-30 \%$ & $-0.323 \pm 0.502$ & $2.534 \pm 0.630$ & 0.072 \\
\hline & & $30-40 \%$ & $-0.452 \pm 0.575$ & $2.864 \pm 0.733$ & 0.111 \\
\hline & & $40-60 \%$ & $-1.016 \pm 0.262$ & $3.391 \pm 0.341$ & 0.020 \\
\hline & & $60-80 \%$ & $-0.001 \pm 0.153$ & $1.966 \pm 0.211$ & 0.011 \\
\hline \multirow[t]{4}{*}{ Figure $7(\mathrm{c})$} & $\kappa-\left\langle p_{T}\right\rangle$ & $7.7 \mathrm{GeV}$ & $-0.221 \pm 0.001$ & $2.666 \pm 0.001$ & 0.001 \\
\hline & & $11.5 \mathrm{GeV}$ & $1.274 \pm 0.342$ & $1.174 \pm 0.379$ & 0.005 \\
\hline & & $19.6 \mathrm{GeV}$ & $-1.353 \pm 0.446$ & $3.990 \pm 0.486$ & 0.008 \\
\hline & & $27 \mathrm{GeV}$ & $-0.715 \pm 0.361$ & $3.511 \pm 0.369$ & 0.019 \\
\hline \multirow[t]{6}{*}{ Figure $7(d)$} & $\kappa-\left\langle p_{T}\right\rangle$ & $\begin{array}{l}39 \text { GeV } \\
0-10 \%\end{array}$ & $\begin{array}{l}-5.062 \pm 1.010 \\
-2.481 \pm 1.539\end{array}$ & $\begin{array}{l}8.021 \pm 1.041 \\
5.294 \pm 1.564\end{array}$ & $\begin{array}{l}0.052 \\
0.148\end{array}$ \\
\hline & & $10-20 \%$ & $-4.378 \pm 0.991$ & $7.308 \pm 1.025$ & 0.035 \\
\hline & & $20-40 \%$ & $-1.980 \pm 0.370$ & $4.726 \pm 0.393$ & 0.026 \\
\hline & & $10-60 \%$ & $1.447 \pm 0.001$ & $0.959 \pm 0.001$ & 0.001 \\
\hline & & $40-60 \%$ & $-1.496 \pm 0.986$ & \pm 1.093 & 0.054 \\
\hline & & $60-80 \%$ & $-4.203 \pm 0.022$ & \pm 0.025 & 0.001 \\
\hline \multirow[t]{5}{*}{ Figure $7(\mathrm{e})$} & $T-\left\langle p_{T}\right\rangle$ & $7.7 \mathrm{GeV}$ & $0.111 \pm 0.007$ & $0.042 \pm 0.008$ & 0.003 \\
\hline & & $11.5 \mathrm{GeV}$ & $0.139 \pm 0.006$ & $0.012 \pm 0.007$ & 0.010 \\
\hline & & $19.6 \mathrm{GeV}$ & $6 \pm 0.011$ & \pm 0.012 & 0.031 \\
\hline & & $27 \mathrm{GeV}$ & $1 \pm 0.016$ & \pm 0.015 & 0.054 \\
\hline & & $39 \mathrm{GeV}$ & $0.140 \pm 0.010$ & \pm 0.008 & 0.011 \\
\hline \multirow[t]{6}{*}{ Figure $7(\mathrm{f})$} & $T-\left\langle p_{T}\right\rangle$ & $0-5 \%$ & $0.154 \pm 0.004$ & $-0.003 \pm 0.004$ & 0.010 \\
\hline & & $5-10 \%$ & $0.155 \pm 0.007$ & $-0.002 \pm 0.006$ & 0.027 \\
\hline & & $10-20 \%$ & $0.143 \pm 0.005$ & $0.009 \pm 0.005$ & 0.017 \\
\hline & & $20-40 \%$ & $0.147 \pm 0.005$ & $0.006 \pm 0.005$ & 0.029 \\
\hline & & $40-60 \%$ & $0.147 \pm 0.007$ & $0.005 \pm 0.008$ & 0.032 \\
\hline & & $60-80 \%$ & $0.145 \pm 0.004$ & $0.004 \pm 0.004$ & 0.010 \\
\hline
\end{tabular}




\section{References}

[1] S. Chatterjee, S. Das, L. Kumar, D. Mishra, B. Mohanty, R. Sahoo, N. Sharma, Adv. High Energy Phys. 2015, 349013 (2015).

[2] Y. Zhong, C.-B. Yang, X. Cai, S.-Q. Feng, Adv. High Energy Phys. 2015, 193039 (2015).

[3] J. Uphoff, O. Fochler, Z. Xu, C. Greiner, Acta Phys. Pol. B Proc. Suppl. 5, 555 (2012).

[4] R. C. Hwa, Adv. High Energy Phys. 2015, 526908 (2015).

[5] G.-L. Ma, M.-W. Nie, Adv. High Energy Phys. 2015, 967474 (2015).

[6] D. D. Ivanenko, D. F. Kurdgelaidze, Astrofizika 1, 479 (1965) [Astrophysics 1, 251 (1965)].

[7] N. Itoh, Prog. Theor. Phys. 44 291, (1970).

[8] T. D. Lee, G. C. Wick, Phys. Rev. D 9, 2291 (1974).

[9] M. Nasim, V. Bairathi, M. K. Sharma, B. Mohanty, A. Bhasin, Adv. High Energy Phys. 2015, 197930 (2015).

[10] For the STAR Collaboration (L. Kumar), Talk given the 2011 Meeting of the Division of Particles and Fields of the American Physical Society (DPF 2011), Rhode Island, USA, August 9-13, 2011, arXiv:1109.5313 (2011).

[11] For the STAR collaboration (S. Das), EPJ Web of Conf. 90, 08007 (2015).

[12] For the STAR collaboration (S. S. Shi), Acta Phys. Pol. B Proc. Suppl. 5, 311 (2012).

[13] For the NA49 NA61/SHINE Collaborations (M. Gazdzicki), J. Phys. G 38, 124024 (2011).

[14] For the NA61/SHINE Collaboration (M. Mackowiak-Pawlowska), PoS (EPS-HEP2015), 205 (2015), arXiv:1510.08688 (2015).

[15] For the NA61 Collaboration (M. Mackowiak), J. Phys. Conf. Ser. 270, 012048 (2011).

[16] For the NA61/SHINE Collaboration (D. T. Larsen), arXiv:1510.00674 (2015).

[17] For the STAR Collaboration (L. Kumar), J. Phys. G 38, 124145 (2011).

[18] A. Rustamov, Cen. Eur. J. Phys. 10, 1267 (2012).

[19] M. Bleicher, arXiv:hep-ph/0509314 (2005).

[20] J. Steinheimer, M. Bleicher, Eur. Phys. J. A 48, 100 (2012).

[21] Y. B. Ivanov, D. Blaschke, Phys. Rev. C 92024916 (2015).

[22] Y. B. Ivanov, Talk given at the 15th International Conference on Strangeness in Quark Matter (SQM2015), Dubna, Russia, 6-11 July 2015, arXiv:1509.06944 (2015).

[23] F.-H. Liu, L.-N. Gao, R. A. Lacey, Adv. High Energy Phys. 2016, 9467194 (2016).

[24] R. A. Lacey, Phys. Rev. Lett. 114, 142301 (2015).

[25] F.-H. Liu, Y.-Q. Gao, T. Tian, B.-C. Li, Eur. Phys. J. A 50, 94 (2014).

[26] F.-H. Liu, J.-S. Li, Phys. Rev. C 78, 044602 (2008).

[27] F.-H. Liu, Nucl. Phys. A 810, 159 (2008).

[28] J. Schwinger, Phys. Rev. 82, 664 (1951).

[29] R.-C. Wang, C.-Y. Wong, Phys. Rev. D 38, 348 (1988).

[30] C.-Y. Wong, Introduction to High Energy Heavy Ion Collisions, World Scientific, Singapore, 1994.

[31] P. Braun-Munzinger, K. Redlich, J. Stachel, in Quark-Gluon Plasma 3, edited by R. C. Hwa, X.-N. Wang (World Scientific, Singapore, 2004), arXiv:nucl-th/0304013 (2004).

[32] C. Tsallis, J. Stat. Phys. 52, 479 (1988).

[33] T. S. Biró, G. Purcsel, K. Ürmössy, Eur. Phys. J. A 40, 325 (2009).

[34] J. Cleymans, D. Worku, Eur. Phys. J. A 48, 160 (2012).

[35] STAR Collaboration (L. Adamczyk et al.), arXiv:1506.07605 (2015).

[36] For the STAR Collaboration (M. V. Tokarev), Talk given at the International Conference "HADRON STRUCTURE'15", Horny Smokovec, Slovak Republic, 29 June - 3 July, 2015, arXiv:1509.06570, (2015).

[37] A. Andronic, P. Braun-Munzinger, J. Stachel, Nucl. Phys. A 834, 237c (2010).

[38] F.-H. Liu, Y.-Q. Gao, H.-R. Wei, Adv. High Energy Phys. 2014, 293873 (2014).

[39] L.-N. Gao, F.-H. Liu, Adv. High Energy Phys. 2016, 1505823 (2016).

[40] H.-R. Wei, Y.-H. Chen, L.-N. Gao, F.-H. Liu, Adv. High Energy Phys. 2014, 782631 (2014). 